\title{
Auslander-Reiten theory in a Krull-Schmidt category
}

\section{Shiping Liu}

Département de mathématiques, Université de Sherbrooke, Sherbrooke, Québec, Canada J1K 2R1, and School of Mathematics, Beijing Normal University, Beijing, China.

E-mail address: shiping.liu@usherbrooke.ca

\begin{abstract}
We first introduce the notion of an Auslander-Reiten sequence in a Krull-Schmidt category. This unifies the notion of an almost split sequence in an abelian category and that of an Auslander-Reiten triangle in a triangulated category. We then define the Auslander-Reiten quiver of a Krull-Schmidt category and describe the shapes of its semi-stable components. The main result generalizes those for an artin algebra and specializes to an arbitrary triangulated categories, in particular to the derived category of bounded complexes of finitely generated modules over an artin algebra of finite global dimension.
\end{abstract}

\section{INTRODUCTION}

Since its introduction in the early seventies; see [3, 4], the AuslanderReiten theory, that is the theory of irreducible morphisms and almost split sequences, has been playing a fundamental role in the modern representation theory of artin algebras. It has also an important impact to many other areas of mathematics such as algebraic geometry and algebraic topology; see, for example, $[1,14,15]$. Indeed, it appears naturally in abelian categories such as the module category of

\footnotetext{
Acknowledgement: The author is supported in part by the Natural Sciences and Engineering Research Council of Canada, and was a guest researcher at the Beijing Normal University financed through the 985-project.
} 
an artin algebra; see [4], additive categories with an exact structure such as the representation category of a bocs; see [7], and triangulated categories such as the derived category of bounded complexes in an abelian category; see $[10,14]$ and cluster categories; see [9]. In order to unify the various existent versions, we shall introduce and study this theory in a general Krull-Schmidt category. The results generalize those, with some new ones, for a module category and are applicable to general abelian categories and triangulated categories. We now outline the content of the paper section by section.

While irreducible morphisms have been thoroughly studied in [6], we introduce in Section 1 the notion of an Auslander-Reiten sequence in a Krull-Schmidt category. Compared with those in categories with an exact structure and those in triangulated categories, the AuslanderReiten sequences in a Krull-Schmidt category behave nicely under taking subcategories and quotients by an admissible ideal; see (1.6). This has an interesting application on extensions between indecomposable modules over an artin algebra; see (2.11). In subsequent papers, we shall provide more applications in problems involving the stable module category of an artin algebra.

In Section 2, we define as usual the Auslander-Reiten quiver of a Krull-Schmidt category. One of the important features of the Auslander-Reiten quiver of an artin algebra is that it does not contain any sectional oriented cycle; see [8]. We shall prove that the same holds true for a left or right Auslander-Reiten category; for definition, see $(2.6)$.

In Section 3, we recall the notion of degrees of an irreducible morphism, which is originally introduced in a module category; see [18]. This is a powerful tool in handling oriented cycles in an AuslanderReiten quiver, the hardest task in describing the shapes of AuslanderReiten components.

Section 4 is purely combinatorial which deals with semi-stable valued translation quivers. If such a valued translation quiver contains no oriented cycle, then it embeds in $\mathbb{Z} \Delta$ with $\Delta$ some valued quiver without oriented cycles. On the other hand, the typical examples of semi-stable valued translations quivers containing oriented cycles are ray tubes and co-ray tubes. A characterization of these semi-stable 
tubes was implicitly used in [19] and was explicitly stated in [20, Section 2] but with no proof. Later, a rigorous proof first appeared in a master thesis [16] written under the author's supervision. Since it has never been published, we include an improved version here.

Section 5 contains the main results of the paper. For many special kinds of categories, a complete description of the Auslander-Reiten components has been obtained; see, for example, [10, 14, 17, 23, 25]. For the module category of an artin algebra, a general description of these components can be found in $[11,19,28]$. Using the theory of degrees of irreducible morphisms as we did in $[18,19]$, we shall show that most of these results hold true for Krull-Schmidt categories, except that loops may show up in general. Fortunately, if the category is a connected left or right Auslander-Reiten category, then we can determine completely the Auslander-Reiten quiver in case it contains a loop.

In Section 6, we specialize our results to a triangulated category which is triangle-connected but not triangle-simple. If such a triangulated category admits a left or right Serre functor; see [22], then each of its Auslander-Reiten components is either a stable or semistable tube, or of shape $\mathbb{Z} \Delta / G$ with $\Delta$ a Dynkin quiver and $G$ a group of automorphisms containing a positive power of the translation, or embeds in $\mathbb{Z} \Delta$ with $\Delta$ a locally finite valued quiver containing no oriented cycle, where the semi-stable tubes will not appear if the functor is a Serre functor. Applying this to the bounded derived category of an artin algebra of finite global dimension, we show that every Auslander-Reiten component is a stable tube or of shape $\mathbb{Z} \Delta$ where $\Delta$ is a locally finite valued quiver with no oriented cycle.

\section{Auslander-Reiten sequences}

All categories considered in this paper are skeletally small. Morphisms in a category are composed from the left to the right. Let $R$ denote a fixed commutative artinian ring. An $R$-category is a category in which the morphism sets are $R$-modules and the composition of morphisms is $R$-bilinear. Such an $R$-category is Hom-finite if the morphism sets are finitely generated as $R$-modules. Further, one says that an idempotent $e: X \rightarrow X$ in an $R$-category splits if there exist 
morphisms $f: X \rightarrow Y$ and $g: Y \rightarrow X$ such that $e=f g$ and $g f=\mathbb{1}_{Y}$. In a Hom-finite $R$-category, it is well known that idempotents split if and only if indecomposable objects have a local endomorphism algebra.

Throughout, $\mathcal{A}$ stands for a Krull-Schmidt $R$-category, that is a Hom-finite additive $R$-category in which idempotents split. The $J a$ cobson radical $\operatorname{rad}(\mathcal{A})$ of $\mathcal{A}$ is the ideal generated by the non-isomorphisms between indecomposable objects. For $n>1, \operatorname{rad}^{n}(\mathcal{A})$ denotes the ideal generated by the morphisms which are composite of $n$ morphisms lying in the radical. Finally, $\operatorname{rad}^{\infty}(\mathcal{A})=\cap_{n \geq 1} \operatorname{rad}^{n}(\mathcal{A})$ is called the infinite radical of $\mathcal{A}$.

Let $f: X \rightarrow Y$ be a morphism in $\mathcal{A}$. Recall from $[4,5]$ that $f$ is irreducible if $f$ is neither a section nor a retraction while every factorization $f=g h$ implies that $g$ is a section or $h$ is a retraction. One calls $f$ a source morphism, originally called minimal left almost split morphism $[4,5]$, if $f$ is not a section such that every non-section morphism $X \rightarrow M$ factors through $f$ and every factorization $f=f h$ implies that $h$ an automorphism of $Y$. In this case, $X$ is indecomposable, and if $f$ is non-zero, then $g: X \rightarrow M$ is irreducible if and only if $g=f h$ with $h: Y \rightarrow M$ a retraction. One defines a sink morphism, originally called minimal right almost split morphism [4, 5], in a dual manner. If $f$ is a sink morphism, then $Y$ is indecomposable, and if $f$ is non-zero, then $g: N \rightarrow Y$ is irreducible if and only if $g=h f$ with $h: N \rightarrow X$ a section.

We begin with two easy results concerning sink morphisms, which are known in a module category; see, for example, [5]. Note that the dual results for source morphisms also hold true.

1.1. Lemma. If $g: Y \rightarrow Z$ is a sink morphism in $\mathcal{A}$, then $g=0$ if and only if $Y=0$.

Proof. If $g: Y \rightarrow Z$ is a zero sink morphism, then $g=0_{Y} g$. Thus $0_{Y}$ is an automorphism of $Y$, that is $Y=0$. The proof of the lemma is completed. 
1.2. LemMA. Given a commutative square

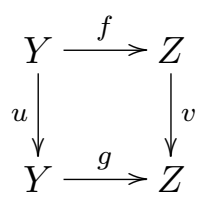

in $\mathcal{A}$, where $f, g$ are non-zero sink morphisms. Then $u$ is an automorphism of $Y$ if and only if $v$ is an automorphism of $Z$.

Proof. Assume that $u$ is an automorphism of $Y$, while $v$ is not an automorphism of $Z$. Since $Z$ is indecomposable, $\operatorname{End}_{\mathcal{A}}(Z)$ is local. Thus $v^{r}=0$ for some $r>0$. This yields $u^{r} g=f v^{r}=0$, and hence $g=0$, a contradiction. Suppose now that $v$ is an automorphism of $Z$. Then $g v^{-1}$ is not a retraction. Hence $g v^{-1}=w f$ for some $w \in \operatorname{End}(Y)$. This gives rise to $f=f(u w)$ and $g=g(w u)$. Hence, $u w$ and $w u$ are automorphisms of $Y$. Therefore, $u$ is an automorphism of $Y$. The proof of the lemma is completed.

Recall that a short exact sequence $0 \rightarrow X \stackrel{u}{\rightarrow} Y \stackrel{v}{\rightarrow} Z \rightarrow 0$ in an abelian category is an almost split sequence if $u$ is a source morphism and $v$ is a sink morphism; see [3, 5]. We shall generalize this notion to a Krull-Schmidt category. Let $X \stackrel{f}{\longrightarrow} Y \stackrel{g}{\longrightarrow} Z$ be a sequence of morphisms in $\mathcal{A}$. One says that $f$ is a pseudo-kernel of $g$ if $\operatorname{Hom}_{\mathcal{A}}(M, X) \stackrel{f_{*}}{\longrightarrow} \operatorname{Hom}_{\mathcal{A}}(M, Y) \stackrel{g_{*}}{\longrightarrow} \operatorname{Hom}_{\mathcal{A}}(M, Z)$ is an exact sequence for every object $M$ in $\mathcal{A}$, and that $g$ is a pseudo-cokernel of $f$ if $\operatorname{Hom}_{\mathcal{A}}(Z, N) \stackrel{g^{*}}{\longrightarrow} \operatorname{Hom}_{\mathcal{A}}(Y, N) \stackrel{f^{*}}{\longrightarrow} \operatorname{Hom}_{\mathcal{A}}(X, N)$ is exact for every $N$ in $\mathcal{A}$. Now we call the sequence $X \stackrel{f}{\longrightarrow} Y \stackrel{g}{\longrightarrow} Z$ short exact if $f$ is the kernel of $g$ while $g$ is the cokernel of $f$, and short pseudo-exact if $f$ is a pseudo-kernel of $g$ while $g$ is a pseudo-cokernel of $f$.

1.3. Definition. A short pseudo-exact sequence $X \stackrel{f}{\longrightarrow} Y \stackrel{g}{\longrightarrow} Z$ in $\mathcal{A}$ with $Y \neq 0$ is called an Auslander-Reiten sequence if $f$ is a source morphism and $g$ is a sink morphism.

It follows from Lemma 1.1 that an Auslander-Reiten sequence contains no zero morphism. This property ensures that the following classical result for almost split sequences in an abelian category holds true in a general Krull-Schmidt category; compare [5]. 
1.4. Theorem. Let $\mathcal{A}$ be a Krull-Schmidt R-category with $X \stackrel{f}{\longrightarrow}$ $Y \stackrel{g}{\longrightarrow} Z$ an Auslander-Reiten sequence.

(1) Up to isomorphism, the sequence is the unique Auslander-Reiten sequence starting with $X$ and the unique one ending with $Z$.

(2) Each irreducible morphism $f_{1}: X \rightarrow Y_{1}$ or $g_{1}: Y_{1} \rightarrow Z$ fits into an Auslander-Reiten sequence

$$
X \stackrel{\left(f_{1}, f_{2}\right)}{\longrightarrow} Y_{1} \amalg Y_{2} \stackrel{\left(\begin{array}{l}
g_{1} \\
g_{2}
\end{array}\right)}{\longrightarrow} Z .
$$

Proof. Let $X \stackrel{f^{\prime}}{\longrightarrow} Y^{\prime} \stackrel{g^{\prime}}{\longrightarrow} Z^{\prime}$ be another Auslander-Reiten sequence in $\mathcal{A}$. By the uniqueness of source morphisms, there exist isomorphisms $u: Y \rightarrow Y^{\prime}$ and $u^{\prime}: Y^{\prime} \rightarrow Y$ such that $f^{\prime}=f u$ and $f=f^{\prime} u^{\prime}$. Now $f\left(u g^{\prime}\right)=f^{\prime} g^{\prime}=0$ and $f^{\prime}\left(u^{\prime} g\right)=f g=0$. Since the sequences are short pseudo-exact, we get a commutative diagram as follows:

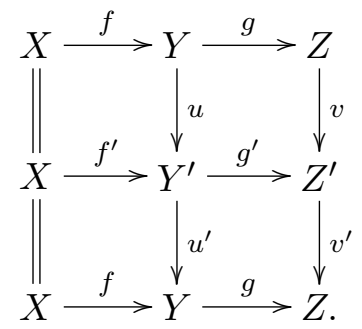

Since $u u^{\prime}$ is an automorphism of $Y$, by Lemma 1.2, $v v^{\prime}$ is an automorphism of $Z$. Similarly, $v^{\prime} v$ is an automorphism of $Z^{\prime}$, and hence $v$ is an isomorphism. This proves the uniqueness for $X$, and dually we have the uniqueness for $Z$.

Now let $f_{1}: X \rightarrow Y_{1}$ be an irreducible morphism in $\mathcal{A}$. Since $f: X \rightarrow Y$ is a source morphism, $f_{1}$ fits in a source morphism $\left(f_{1}, f_{2}\right)$ : $X \rightarrow Y_{1} \amalg Y_{2}$. Hence there exists an isomorphism $u: Y \rightarrow Y_{1} \amalg Y_{2}$ such that $\left(f_{1}, f_{2}\right)=f u$. Setting $\left(\begin{array}{l}g_{1} \\ g_{2}\end{array}\right)=u^{-1} g$, we get the following commutative diagram :

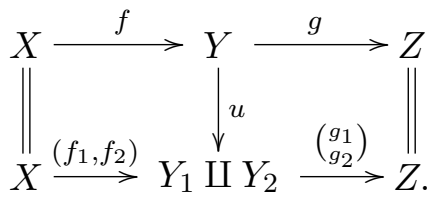


Since $u$ is an isomorphism, the lower row is an Auslander-Reiten sequence. Dually, each irreducible morphism $g_{1}: Y_{1} \rightarrow Z$ fits in a desired Auslander-Reiten sequence. The proof of the theorem is completed.

1.5. Proposition. If $\mathcal{A}$ is abelian, then a sequence $X \stackrel{f}{\longrightarrow} Y \stackrel{g}{\longrightarrow}$ $Z$ in $\mathcal{A}$ is an Auslander-Reiten sequence if and only if $0 \rightarrow X \stackrel{f}{\longrightarrow}$ $Y \stackrel{g}{\longrightarrow} Z \rightarrow 0$ is an almost split sequence.

Proof. Let $\mathcal{A}$ be abelian with an Auslander-Reiten sequence $X \stackrel{f}{\longrightarrow}$ $Y \stackrel{g}{\longrightarrow} Z$. Let $h: Y \rightarrow M$ be the cokernel of $f$. Then $h=g u$ and $g=h v$ for some morphisms $u: Z \rightarrow M$ and $v: Z \rightarrow M$. Since $h=h v u$, we get $v u=\mathbb{1}_{M}$ and $u v$ is an idempotent in $\operatorname{End}_{\mathcal{A}}(Z)$. If $u v=0$, then $g=g u v=0$, which is absurd. Since $\operatorname{End}_{\mathcal{A}}(Z)$ is local, $v u=\mathbb{1}_{Z}$. This shows that $g$ is also a cokernel of $f$. Dually, $f$ is a kernel of $g$. The proof of the proposition is completed.

Next, we shall investigate how Auslander-Reiten sequences behave under taking quotients of $\mathcal{A}$. Let $\mathcal{I}$ be an ideal of $\mathcal{A}$. One defines the quotient $\mathcal{A} / I$ of $\mathcal{A}$ modulo $\mathcal{I}$ as follows. The objects of $\mathcal{A} / I$ are those of $\mathcal{A}$, and the morphisms are given by $\operatorname{Hom}_{\mathcal{A} / I}(X, Y)=$ $\operatorname{Hom}_{\mathcal{A}}(X, Y) / \mathcal{I}(X, Y)$. It is evident that $\mathcal{A} / I$ is an additive Homfinite $R$-category. If $X$ is an indecomposable object in $\mathcal{A}$, then either $1_{X} \in \mathcal{I}(X, X)$ or $\mathcal{I}(X, X) \subseteq \operatorname{rad}(X, X)$. Hence $X$, as an object in $\mathcal{A} / I$, is either null or indecomposable with a local endomorphism algebra. Hence $\mathcal{A} / I$ is also a Krull-Schmidt $R$-category.

1.6. Definition. An ideal $\mathcal{I}$ of $\mathcal{A}$ is called admissible provided that it satisfies the following conditions.

(1) If $X, Y$ are indecomposable objects in $\mathcal{A}$ with $1_{X} \notin \mathcal{I}(X, X)$ and $1_{Y} \notin \mathcal{I}(Y, Y)$, then $\mathcal{I}(X, Y) \subseteq \operatorname{rad}^{2}(X, Y)$.

(2) If $f: X \rightarrow Y$ is a source morphism in $\mathcal{A}$ with $1_{X} \notin \mathcal{I}(X, X)$, then every $g \in \mathcal{I}(X, M)$ can be written as $g=f h$ with $h \in \mathcal{I}(Y, M)$.

(3) If $f: X \rightarrow Y$ is a sink morphism in $\mathcal{A}$ with $1_{Y} \notin \mathcal{I}(Y, Y)$, then every $g \in \mathcal{I}(M, Y)$ can be written as $g=h f$ with $h \in \mathcal{I}(M, X)$.

1.7. Lemma. Let $\mathcal{I}$ be an admissible ideal $\mathcal{A}$. For $f \in \operatorname{Hom}_{\mathcal{A}}(X, Y)$, write $\bar{f}=f+\mathcal{I}(X, Y) \in \operatorname{Hom}_{\mathcal{A} / I}(X, Y)$. 
(1) If $f: X \rightarrow Y$ is an irreducible morphism in $\mathcal{A}$ with $X, Y$ indecomposable, then $\bar{f}: X \rightarrow Y$ is irreducible in $\mathcal{A} / I$ whenever $X, Y$ are non-zero in $\mathcal{A} / I$.

(2) If $f: X \rightarrow Y$ is a source (epi)morphism in $\mathcal{A}$, then $\bar{f}: X \rightarrow Y$ is a source (epi)morphism in $\mathcal{A} / I$ whenever $X$ is non-zero in $\mathcal{A} / I$.

(3) If $f: X \rightarrow Y$ is a sink (mono) morphism in $\mathcal{A}$, then $\bar{f}: X \rightarrow Y$ is a sink (mono)morphism in $\mathcal{A} / I$ whenever $Y$ is non-zero in $\mathcal{A} / I$.

Proof. Firstly, let $X, Y$ be indecomposable objects in $\mathcal{A}$ with $1_{X} \notin$ $\mathcal{I}(X, X)$ and $1_{Y} \notin \mathcal{I}(Y, Y)$. By definition, $\mathcal{I}(X, Y) \subseteq \operatorname{rad}^{2}(X, Y)$. This gives rise to

$$
\operatorname{rad}_{\mathcal{A} / I}(X, Y)=\operatorname{rad}(X, Y) / \mathcal{I}(X, Y),
$$

and

$$
\operatorname{rad}_{\mathcal{A} / I}^{2}(X, Y)=\operatorname{rad}^{2}(X, Y) / \mathcal{I}(X, Y) .
$$

Hence, if $f \in \operatorname{rad}(X, Y) \backslash \operatorname{rad}^{2}(X, Y)$, then

$$
\bar{f} \in \operatorname{rad}_{\mathcal{A} / I}(X, Y) \backslash \operatorname{rad}_{\mathcal{A} / I}^{2}(X, Y) .
$$

Next, let $f: X \rightarrow Y$ be a source morphism in $\mathcal{A}$ with $1_{X} \notin \mathcal{I}(X, X)$. We claim that $\bar{f}$ is a not section. If this not the case, then there exists a morphism $f^{\prime}: Y \rightarrow X$ in $\mathcal{A}$ such that $1_{X}-f f^{\prime} \in \mathcal{I}(X, X)$. However, $1_{X}-f f^{\prime}$ is invertible since $f \in \operatorname{rad}(X, Y)$. Hence $1_{X} \in \mathcal{I}(X, X)$, a contradiction. Let $h: X \rightarrow M$ be a morphism in $\mathcal{A}$ such that $\bar{h}$ is not a section. Then $h$ is not a section, and hence factors through $f$. As a consequence, $\bar{h}$ factors through $\bar{f}$. Moreover, let $u: Y \rightarrow Y$ be a morphism in $\mathcal{A}$ such that $\bar{f}=\bar{f} \bar{u}$. Then $f-f u \in \mathcal{I}(X, Y)$. By definition, $f-f u=f v$ with $v \in \mathcal{I}(Y, Y)$. This yields $f=f w$ with $w=u+v$. Then $w$ is an automorphism of $Y$ in $\mathcal{A}$, and hence $\bar{u}=\bar{w}$ is an automorphism of $Y$ in $\mathcal{A} / I$. This shows that $\bar{f}$ is a source morphism. Assume now that $f$ is an epimorphism. If $q: Y \rightarrow N$ is a morphism in $\mathcal{A}$ such that $f q \in \mathcal{I}(X, N)$, then $f q=f q^{\prime}$ with $q^{\prime} \in \mathcal{I}(Y, N)$. Since $f$ is an epimorphism, we have $q=q^{\prime}$. Thus $\bar{f}$ is an epimorphism. This proves Statement (2). In a dual manner, one can prove Statement (3). The proof of the lemma is completed.

1.8. Proposition. Let $\mathcal{I}$ be an admissible ideal of $\mathcal{A}$. If $X \stackrel{f}{\longrightarrow}$ $Y \stackrel{g}{\longrightarrow} Z$ is an Auslander-Reiten sequence in $\mathcal{A}$, then $X \stackrel{\bar{f}}{\longrightarrow} Y \stackrel{\bar{g}}{\longrightarrow}$ 
$Z$ is a pseudo-exact sequence in $\mathcal{A} / I$, which is an Auslander-Reiten sequence whenever $X, Y$, and $Z$ are non-zero in $\mathcal{A} / I$.

Proof. Let $X \stackrel{f}{\longrightarrow} Y \stackrel{g}{\longrightarrow} Z$ be an Auslander-Reiten sequence in $\mathcal{A}$. If $h: Y \rightarrow M$ is a morphism in $\mathcal{A}$ such that $\bar{f} \bar{h}=\overline{0}$ then, since $\mathcal{I}$ is admissible, there exists some $h_{1} \in \mathcal{I}(Y, M)$ such that $f h=f h_{1}$, that is $f\left(h-h_{1}\right)=0$. Thus $h-h_{1}=g h_{2}$ for some $h_{2}: Z \rightarrow M$. As a consequence, $\bar{h}=\bar{g} \bar{h}_{2}$. This shows that $\bar{g}$ is a pseudo-cokernel of $\bar{f}$. Dually, $\bar{f}$ is a pseudo-kernel of $\bar{g}$. If $X, Y, Z$ are all non-zero in $\mathcal{A} / I$, it follows from Lemma 1.7 that $X \stackrel{\bar{f}}{\longrightarrow} Y \stackrel{\bar{g}}{\longrightarrow} Z$ is an Auslander-Reiten sequence in $\mathcal{A} / I$. The proof of the proposition is completed.

We conclude this section with two examples of admissible ideals which are important in our future application.

1.9. Lemma. Let $\mathcal{A}$ be a Krull-Schmidt R-category.

(1) The infinite radical of $\mathcal{A}$ is an admissible ideal.

(2) If $\mathcal{B}$ is a subcategory of $\mathcal{A}$ closed under summands, then the ideal of the morphisms factoring through objects in $\mathcal{B}$ is admissible.

Proof. (1) The condition stated in Definition 1.6(1) is clearly satisfied. Let $f: X \rightarrow Y$ be a source morphism in $\mathcal{A}$, and let $g: X \rightarrow M$ be a morphism lying in the infinite radical. Note that $\operatorname{rad}^{\infty}(Y, M)=$ $\operatorname{rad}^{r}(Y, M)$ for some $r>0$. Write $g=\sum_{i=1}^{s} g_{i 0} g_{i 1} \cdots g_{i r}$, where the $g_{i j}$ lie in the radical. Then $g_{i 0}=f h_{i}, i=1, \ldots, s$. Hence $g=f h$, where $h=\sum_{i=1}^{s} h_{i} g_{i 1} \cdots g_{i r} \in \operatorname{rad}^{r}(Y, M)=\operatorname{rad}^{\infty}(Y, M)$. This verifies Definition 1.6(2), and one can verify Definition 1.6(3) in a dual manner.

(2) Let $\mathcal{B}$ be a subcategory of $\mathcal{A}$ closed under summands, and let $\mathcal{I}$ be the ideal of the morphisms factoring through objects in $\mathcal{B}$. Let $X, Y$ be indecomposable objects in $\mathcal{A}$ not isomorphic to any objects in $\mathcal{B}$. If $f \in \mathcal{I}(X, Y)$, then $f=u v$, where $u: X \rightarrow L$ and $v: L \rightarrow Y$ are morphisms with $L \in \mathcal{B}$. Since $\mathcal{B}$ is closed under summands, $u$ is not a section and $v$ is not a retraction. Hence $f \in \operatorname{rad}^{2}(X, Y)$. This verifies Definition 1.6(1). Let now $g: X \rightarrow Y$ be a source morphism in $\mathcal{A}$ with $X$ not isomorphic to any object in $\mathcal{B}$. If $h \in \mathcal{I}(X, M)$, then $h=h_{1} h_{2}$, where $h_{1}: X \rightarrow N$ and $h_{2}: N \rightarrow M$ are morphisms with $N \in \mathcal{B}$. Note that $h_{1}$ is not a section. Hence $h_{1}=f h_{3}$ for some $h_{3}: Y \rightarrow N$. This yields $h=f\left(h_{3} h_{2}\right)$, where $h_{3} h_{2}$ factors through $N$. 
Thus Definition 1.6(2) is verified. Dually, one can verify Definition 1.6(3). The proof of the lemma is completed.

\section{The Auslander-Reiten Quiver}

The objective of this section is to define the Auslander-Reiten quiver of a Krull-Schmidt category. We begin with a brief recall of some combinatorial background. Firstly, a quiver $\Gamma$ consists of a set $\Gamma_{0}$ of vertices and a set $\Gamma_{1}$ of arrows between vertices. If $x \rightarrow y$ is an arrow in $\Gamma$, we say that $x$ is an immediate predecessor of $y$ while $y$ is an immediate successor of $x$. For a vertex $x$ in $\Gamma$, the set of immediate predecessors of $x \Gamma$ and that of immediate successors are denoted by $x^{+}$and $x^{-}$, respectively. We say that $\Gamma$ is left (respectively, right) locally finite if $x^{-}$(respectively, $x^{+}$) is finite for all $x \in \Gamma_{0}$, and locally finite if it is left and right locally finite. A subquiver $\Sigma$ of a quiver $\Gamma$ is called full if all arrows $x \rightarrow y$ in $\Gamma$ with $x, y \in \Sigma_{0}$ lie in $\Sigma$, and convex if every path in $\Gamma$ with its end-points lying in $\Sigma$ lies entirely in $\Sigma$.

Next, a valued quiver consists of a pair $(\Gamma, v)$, where $\Gamma$ is a quiver without multiple arrows and $v$ is a valuation for the arrows, that is, each arrow $x \rightarrow y$ is endowed with a pair of positive integers $\left(v_{x y}, v_{x y}^{\prime}\right)$. We say that an arrow $x \rightarrow y$ has trivial valuation if $v_{x y}=v_{x y}^{\prime}=1$, and $\Gamma$ has trivial valuation if all arrows have trivial valuation. A valued quiver $(\Sigma, u)$ is a valued subquiver of $(\Gamma, v)$ if $\Sigma$ is a subquiver of $\Gamma$ in which the arrows have the same valuation as in $\Gamma$.

Moreover, a valued translation quiver consists of a triple $(\Gamma, \rho, v)$, where $(\Gamma, v)$ is a valued quiver, and $\rho$ is a bijection, called the translation, from one subset of $\Gamma_{0}$ to another one such that if $x$ is a vertex with $\rho x$ defined, then $x^{+}=(\rho x)^{-} \neq \emptyset$ and $\left(v_{\rho x, y}, v_{\rho x, y}^{\prime}\right)=\left(v_{y x}^{\prime}, v_{y x}\right)$ for every $y \in x^{+}$. We should point out that valued translation quivers considered in this paper are not necessarily locally finite and may contain loops; compare [11, Section 2]. A vertex $x$ in $(\Gamma, \rho, v)$ is called projective or injective if $\rho x$ or $\rho^{-} x$ is not defined, respectively. Finally, a valued translation quiver $(\Sigma, \sigma, u)$ is a valued translation subquiver of $(\Gamma, \rho, v)$ if $(\Sigma, u)$ is a valued subquiver of $(\Gamma, v)$ such that each non-projective vertex $x$ in $\Sigma$ is not projective in $\Gamma$ and $\sigma x=\rho x$. 
We now turn our attention back to a Krull-Schmidt $R$-category $\mathcal{A}$. If $X, Y$ are indecomposable objects in $\mathcal{A}$, then $\operatorname{irr}(X, Y)=\operatorname{rad}(X, Y) /$ $\operatorname{rad}^{2}(X, Y)$ is a $k_{X}-k_{Y}$-bimodule, where $k_{Z}=\operatorname{End}(Z) / \operatorname{rad}(Z, Z)$. The dimension of $\operatorname{irr}(X, Y)$ over $k_{X}$ and that over $k_{Y}$ are written as $d_{X Y}^{\prime}$ and $d_{X Y}$, respectively. It follows from Bautista's result in $[6,(3.4)]$ that if $\operatorname{irr}(X, Y) \neq 0$, then $d_{X Y}^{\prime}$ is the maximal integer such that $\mathcal{A}$ admits an irreducible morphism from $X^{d_{X Y}^{\prime}}$ to $Y$, while $d_{X Y}$ is the maximal integer such that $\mathcal{A}$ admits an irreducible morphism from $X$ to $Y^{d_{X Y}}$, where $Z^{n}$ denotes the co-product of $n$ copies of $Z$. As a consequence, if $M \rightarrow Y$ is a sink morphism, then $d_{X Y}^{\prime}$ is the multiplicity of $X$ as a summand of $M$, and if $X \rightarrow N$ is a source morphism, then $d_{X Y}$ is the multiplicity of $Y$ as a summand of $N$.

We are ready to define the Auslander-Reiten quiver $\Gamma_{\mathcal{A}}$ of $\mathcal{A}$ as follows. The vertex set is a complete set of representatives of the isomorphism classes of indecomposable objects in $\mathcal{A}$. For vertices $X, Y$, there exists an unique arrow $X \rightarrow Y$ with valuation $\left(d_{X Y}, d_{X Y}^{\prime}\right)$ if and only if $\operatorname{irr}(X, Y) \neq 0$. The translation $\tau$, called the AuslanderReiten translation, is such that $X=\tau Z$ if and only if $\mathcal{A}$ has an Auslander-Reiten sequence $X \rightarrow Y \rightarrow Z$.

2.1. Proposition. If $\mathcal{A}$ is a Krull-Schmidt $R$-category, then the Auslander - Reiten quiver $\Gamma_{\mathcal{A}}$ of $\mathcal{A}$ is a valued translation quiver.

Proof. Let $\mathcal{A}$ be a Krull-Schmidt $R$-category, and let $Y$ be an object in $\Gamma_{\mathcal{A}}$ with $\tau Y$ defined. Then $\mathcal{A}$ has an Auslander-Reiten sequence $\tau Y \stackrel{f}{\longrightarrow} M \stackrel{g}{\longrightarrow} Y$. Since $M$ is non-zero, $Y$ has at least one immediate predecessor in $\Gamma_{\mathcal{A}}$. Now let $X \rightarrow Y$ be an arrow in $\Gamma_{\mathcal{A}}$. Then $X$ is a summand of $M$. Since $f$ is a source morphism, $\mathcal{A}$ admits an irreducible morphism from $\tau Y$ to $X$, that is $\Gamma_{\mathcal{A}}$ contains an arrow $\tau Y \rightarrow X$. This shows that $\tau$ is a translation for $\Gamma_{\mathcal{A}}$. Moreover, both $d_{X Y}^{\prime}$ and $d_{\tau Y, X}$ are equal to the multiplicity of $X$ as a summand of $M$. It remains to show that $d_{\tau Y, X}^{\prime}=d_{X Y}$. Indeed, by definition, each $h \in \operatorname{End}_{\mathcal{A}}(Y)$ induces a commutative diagram

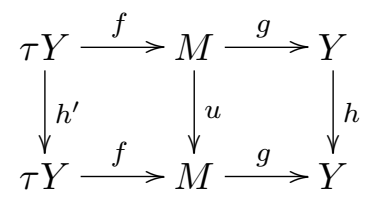


in $\mathcal{A}$, and every $h^{\prime} \in \operatorname{End}(\tau Y)$ can be induced in this way. Applying Lemma 1.2 and its dual, we see that $h$ is an automorphism of $Y$ if and only if $h^{\prime}$ is an automorphism of $\tau Y$. This shows that $k_{Y} \cong$ $k_{\tau Y}$ as $R$-algebras. In particular, $l_{R}\left(k_{Y}\right)=l_{R}\left(k_{\tau Y}\right)$, where $l_{R}(U)$ denotes the $R$-length of a finitely generated $R$-module $U$. Therefore, $l_{R}(\operatorname{irr}(\tau Y, X))=l_{R}\left(k_{\tau Y}\right) d_{\tau Y, X}^{\prime}=l_{R}\left(k_{Y}\right) d_{\tau Y, X}^{\prime}$. On the other hand, one has

$$
\begin{gathered}
l_{R}(\operatorname{irr}(\tau Y, X))=l_{R}\left(k_{X}\right) d_{\tau Y, X}=l_{R}\left(k_{X}\right) d_{X Y}^{\prime}= \\
=l_{R}(\operatorname{irr}(X, Y))=l_{R}\left(k_{Y}\right) d_{X, Y} .
\end{gathered}
$$

This yields $d_{\tau Y, X}^{\prime}=d_{X Y}$. The proof of the proposition is completed.

2.2. Definition. An object $X$ in $\mathcal{A}$ is called pseudo-projective if there exists a sink monomorphism $M \rightarrow X$, and dually, pseudoinjective if there exists a source epimorphism $X \rightarrow N$.

2.3. Lemma. If $X$ is a pseudo-projective object in $\mathcal{A}$, then there exists no Auslander-Reiten sequence ending with $X$. Dually, if $X$ is pseudo-injective, then there exists no Auslander-Reiten sequence starting with $X$.

Proof. Assume that $\mathcal{A}$ admits an Auslander-Reiten sequence $Z \stackrel{f}{\longrightarrow}$ $Y \stackrel{g}{\longrightarrow} X$ as well as a sink monomorphism $h: M \rightarrow X$. Then $g=u h$, where $u$ is an isomorphism. In particular, $g$ is a monomorphism. Hence $f=0$, a contradiction. The proof of the lemma is completed.

2.4. Proposition. If $\mathcal{A}$ is abelian, then an object $X$ in $\mathcal{A}$ is pseudo-projective if and only if $X$ is indecomposable projective with a unique maximal subobject, and $X$ is pseudo-injective if and only if $X$ is indecomposable injective with a unique maximal quotient object.

Proof. Let $\mathcal{A}$ be abelian with $X$ an indecomposable object. It suffices to prove the first part of the lemma, for which the sufficiency is well-known; see, for example, [22]. Suppose that $\mathcal{A}$ has a sink monomorphism $f: M \rightarrow X$. If $g: N \rightarrow X$ is a monomorphism which is not an isomorphism, then $g$ is not an epimorphism. Hence $g=u f$ for some morphism $u: N \rightarrow M$. This shows that $M$ is the unique maximal subobject of $X$. If there exists an epimorphism $h: L \rightarrow X$ which is not a retraction, then $h=v f$ for some $v: L \rightarrow M$. In particular, $f$ is an epimorphism, and hence an isomorphism. This 
contradiction shows that $X$ is projective. The proof of the proposition is completed.

The following easy result will play an important role in our investigation. It was first formulated in a module category $[18,(1.2)]$, and its validity in $\mathcal{A}$ can be established by an easy adaption of the argument given in $[20,(1.4)]$.

2.5. Lemma. Let $f: Y \rightarrow Z$ be an irreducible morphism in $\mathcal{A}$ with $Z$ indecomposable. If there exists $\theta: M \rightarrow Y$ with $\theta \in$ $\operatorname{rad}^{n}(M, Y) \backslash \operatorname{rad}^{n+1}(M, Y)$ for some $n>0$ such that $\theta f \in \operatorname{rad}^{n+2}(M, Z)$, then

(1) $Z$ is not pseudo-projective, and

(2) if $\mathcal{A}$ admits an Auslander-Reiten sequence

$$
X \stackrel{\left(f^{\prime}, g^{\prime}\right)}{\longrightarrow} Y \amalg Y^{\prime} \stackrel{\left(\begin{array}{l}
f \\
g
\end{array}\right)}{\longrightarrow} Z,
$$

then there exists $\eta: M \rightarrow X$ such that $\eta \notin \operatorname{rad}^{n}(M, X), \theta+\eta f^{\prime} \in$ $\operatorname{rad}^{n+1}(M, Y)$, and $\eta g^{\prime} \in \operatorname{rad}^{n+1}\left(M, Y^{\prime}\right)$.

2.6. Definition. A Krull-Schmidt $R$-category $\mathcal{A}$ is called a left Auslander-Reiten category if each indecomposable object in $\mathcal{A}$ is either pseudo-projective or the end-term of an Auslander-Reiten sequence; a right Auslander-Reiten category if each indecomposable object in $\mathcal{A}$ is either pseudo-injective or the starting term of an AuslanderReiten sequence; and an Auslander-Reiten category if it is a left and right Auslander-Reiten category.

REMARK. It is easy to see that if $\mathcal{A}$ is a left or right AuslanderReiten category, then $\Gamma_{\mathcal{A}}$ is left or right locally finite, respectively.

ExAmple. (1) If $A$ is an artin algebra then $\bmod A$, the category of finitely generated right $A$-modules, is an Auslander-Reiten category; see $[3,5]$.

(2) If $k$ is an algebraically closed field, then the category of coherent sheaves over $\mathbf{P}^{n}(k)$ with $n>1$ can be exhausted an ascending chain of left Auslander-Reiten subcategories; see [21]. 
(3) The finite dimensional representations of the infinite quiver

$$
\cdots \rightarrow n \rightarrow \cdots \rightarrow 2 \rightarrow 1
$$

over a field form a right Auslander-Reiten category; see [22].

Let $p: x_{0} \rightarrow x_{1} \rightarrow \cdots \rightarrow x_{n}$ with $n>0$ be a path in a valued translation quiver $(\Gamma, \rho, v)$. A hook in $p$ is an index $i$ with $0<i<n$ such that $x_{i-1}=\rho x_{i+1}$. We say that $p$ is sectional if $p$ admits no hook; and pre-sectional if, for each hook $i$ in $p$, one has $v_{x_{i}, x_{i+1}}>1$, or equivalently $v_{x_{i-1}, x_{i}}^{\prime}>1$. Clearly, a sectional path is pre-sectional. Furthermore, an oriented cycle $x_{0} \rightarrow x_{1} \rightarrow \cdots \rightarrow x_{n}=x_{0}$ is called sectional or pre-sectional if the augmented path

$$
x_{0} \rightarrow x_{1} \rightarrow \cdots \rightarrow x_{n-1} \rightarrow x_{0} \rightarrow x_{1}
$$

is sectional or pre-sectional, respectively. Specializing to the AuslanderReiten quiver $\Gamma_{\mathcal{A}}$, note that a path $X_{0} \rightarrow X_{1} \rightarrow \cdots \rightarrow X_{n}$ is presectional if and only if, for each hook $i$, there exists an irreducible morphism $X_{i-1} \amalg X_{i-1} \rightarrow X_{i}$, or equivalently an irreducible morphism $X_{i} \rightarrow X_{i+1} \amalg X_{i+1}$, in $\mathcal{A}$.

2.7. Lemma. Let $\mathcal{A}$ be a left or right Auslander-Reiten category, and let $X_{0} \rightarrow X_{1} \rightarrow \cdots \rightarrow X_{n}$ with $n>0$ be a path in $\Gamma_{\mathcal{A}}$.

(1) If the path is sectional and $f_{i}: X_{i-1} \rightarrow X_{i}$ is irreducible, $i=$ $1, \ldots, n$, then $f_{1} \cdots f_{n} \notin \operatorname{rad}^{n+1}\left(X_{0}, X_{n}\right)$.

(2) If the path is pre-sectional, then $\mathcal{A}$ admits some irreducible morphisms $f_{i}: X_{i-1} \rightarrow X_{i}, i=1, \ldots, n$, such that $f_{1} \cdots f_{n} \notin$ $\operatorname{rad}^{n+1}\left(X_{0}, X_{n}\right)$.

Proof. We consider only the case where $\mathcal{A}$ is a left Auslander-Reiten category. For $i=2, \ldots, n$, set $Y_{i}=\tau X_{i}$ if $X_{i}$ is not pseudo-projective and $Y_{i}=0$ otherwise, and put $Y_{n+1}=0$. In both cases, $\mathcal{A}$ has irreducible morphisms from $X_{i-1} \amalg Y_{i+1}$ to $X_{i}, i=1, \ldots, n$.

Assume first that the path is sectional. Let $f_{i}: X_{i-1} \rightarrow X_{i}, i=$ $1, \ldots, n$, be irreducible morphisms. Applying Lemma 2.5, one can show by induction that if $\left(\begin{array}{l}f_{i} \\ g_{i}\end{array}\right): X_{i-1} \amalg Y_{i+1} \rightarrow X_{i}$ is irreducible, then $f_{1} \cdots f_{i}-\eta_{i} g_{i} \notin \operatorname{rad}^{i+1}\left(X_{0}, X_{i}\right)$, for every $\eta_{i}: X_{0} \rightarrow Y_{i+1}$; for details, see $[12,(13.3)]$. Setting $\eta_{n}=0$, we get $f_{1} \cdots f_{n} \notin \operatorname{rad}^{n+1}\left(X_{0}, X_{n}\right)$. 
Assume next that the path is pre-sectional. Applying Lemma 2.5, one can find by induction irreducible morphisms $\left(\begin{array}{l}f_{i} \\ g_{i}\end{array}\right): X_{i-1} \amalg Y_{i+1} \rightarrow$ $X_{i}, i=1, \ldots, n$, such that $f_{1} \cdots f_{i}-\eta_{i} g_{i} \notin \operatorname{rad}^{i+1}\left(X_{0}, X_{i}\right)$ for every $\eta_{i}: X_{0} \rightarrow Y_{i+1}$; for details, see [18, (1.15)]. The proof of the lemma is completed.

The following theorem generalizes a well known result of Bautista and Smalø saying that the Auslander-Reiten quiver of an artin algebra has no sectional oriented cycle; see [8].

2.8. TheOREM. If $\mathcal{A}$ is a left or right Auslander-Reiten category, then $\Gamma_{\mathcal{A}}$ contains no pre-sectional oriented cycle.

Proof. We consider only the case where $\mathcal{A}$ is a left AuslanderReiten category. Let $X_{0} \rightarrow \cdots \rightarrow X_{n-1} \rightarrow X_{n}=X_{0}$ be an oriented cycle in $\Gamma_{\mathcal{A}}$. Suppose that $X_{0} \rightarrow \cdots \rightarrow X_{n-1} \rightarrow X_{0} \rightarrow X_{1}$ is a pre-sectional path. Let $r$ be the nilpotency of the radical of $\operatorname{End}\left(X_{1}\right)$. Repeating the preceding path, we get a pre-sectional path $Y_{0} \rightarrow \cdots \rightarrow$ $Y_{n-1} \rightarrow Y_{n} \rightarrow \cdots \rightarrow Y_{r n-1} \rightarrow Y_{r n}$ in $\Gamma_{\mathcal{A}}$, where $Y_{j n+i}=X_{i}, 0 \leq$ $j \leq r$ and $0 \leq i<n$. By Lemma 2.7(2), there exist irreducible morphisms $f_{i}: Y_{i-1} \rightarrow Y_{i}$ such that $f_{1} \cdots f_{r n} \neq 0$. On the other hand, $f_{1} \cdots f_{r n} \in \operatorname{rad}^{r}\left(X_{1}, X_{1}\right)=0$, a contradiction. The proof of the theorem is completed.

Next, we shall study the quotient categories of a left or right Auslander-Reiten category modulo an admissible ideal.

2.9. Proposition. Let $\mathcal{I}$ be an admissible ideal of $\mathcal{A}$. If $\mathcal{A}$ is $a$ (left, right) Auslander-Reiten category, then $\mathcal{A} / \mathcal{I}$ is a (left, right) Auslander-Reiten category, and $\Gamma_{\mathcal{A} / I}$ is the full translation subquiver of $\Gamma_{\mathcal{A}}$ generated by the objects $X$ with $1_{X} \notin \mathcal{I}$.

Proof. We consider only the case where $\mathcal{A}$ is a right AuslanderReiten category. First of all, the objects $X$ in $\Gamma_{\mathcal{A}}$ with $1_{X} \notin \mathcal{I}$ form a complete set of representatives of the isomorphism classes of indecomposable objects in $\mathcal{A} / I$. Fix an object $X$ in $\Gamma_{\mathcal{A}}$ with $1_{X} \notin \mathcal{I}$. If $X$ is pseudo-injective in $\mathcal{A}$ then, by Lemma $1.7(2), X$ is pseudoinjective in $\mathcal{A} / I$. Otherwise, $\mathcal{A}$ has an Auslander-Reiten sequence $X \stackrel{f}{\longrightarrow} Y \stackrel{g}{\longrightarrow} Z$. By Proposition 1.8, $X \stackrel{\bar{f}}{\longrightarrow} Y \stackrel{\bar{g}}{\longrightarrow} Z$ is short pseudoexact in $\mathcal{A} / I$, where $\bar{f}$ is a source morphism by Lemma $1.7(2)$. If 
$1_{Y} \in \mathcal{I}$ or $1_{Z} \in \mathcal{I}$, then it is easy to see that $\bar{f}$ is an epimorphism, that is $X$ is pseudo-injective in $\mathcal{A} / I$. If $1_{Y} \notin \mathcal{I}$ and $1_{Z} \notin \mathcal{I}$ then, by Proposition 1.9, $X \stackrel{\bar{f}}{\longrightarrow} Y \stackrel{\bar{g}}{\longrightarrow} Z$ is an Auslander-Reiten sequence in $\mathcal{A} / I$. Thus, $\mathcal{A} / I$ is a right Auslander-Reiten category.

Next, if $Y$ is another object in $\Gamma_{\mathcal{A}}$ with $1_{Y} \notin \mathcal{I}$ then, as we have seen before, $\operatorname{rad}_{\mathcal{A} / I}(X, Y)=\operatorname{rad}(X, Y) / \mathcal{I}(X, Y)$ and $\operatorname{rad}_{\mathcal{A} / I}^{2}(X, Y)=$ $\operatorname{rad}^{2}(X, Y) / \mathcal{I}(X, Y)$. As a consequence, we have $\operatorname{irr}_{\mathcal{A} / I}(X, Y) \cong$ $\operatorname{irr}(X, Y)$ as $R$-modules. Moreover, $\bar{k}_{X}=\operatorname{End}_{\mathcal{A} / I}(X) / \operatorname{rad}_{\mathcal{A} / I}(X, X) \cong$ $k_{X}$, and $\bar{k}_{Y}=\operatorname{End}_{\mathcal{A} / I}(Y) / \operatorname{rad}_{\mathcal{A} / I}(Y, Y) \cong k_{Y}$ as $R$-algebras. This concludes that $\Gamma_{\mathcal{A} / I}$ contains an arrow $X \rightarrow Y$ if and only if so does $\Gamma_{\mathcal{A}}$, and in this case, $X \rightarrow Y$ has the same valuation in $\Gamma_{\mathcal{A} / I}$ and in $\Gamma_{\mathcal{A}}$. Finally, let $\mathcal{A} / I$ have an Auslander-Reiten sequence $M \rightarrow N \rightarrow X$. We may assume that $M, N \in \Gamma_{\mathcal{A}}$ with $1_{M} \notin \mathcal{I}$ and $1_{N} \notin \mathcal{I}$. As seen previously, $\mathcal{A}$ has an Auslander-Reiten sequence $M \stackrel{u}{\longrightarrow} U \stackrel{v}{\longrightarrow} V$, where $U, V \in \Gamma_{\mathcal{A}}$ with $1_{U} \notin \mathcal{I}$ and $1_{V} \notin \mathcal{I}$, which induces an AuslanderReiten sequence $M \stackrel{\bar{u}}{\longrightarrow} U \stackrel{\bar{v}}{\longrightarrow} V$ in $\mathcal{A} / I$. By Theorem 1.4(1), $X=V$. Hence the Auslander-Reiten translate of $X$ in $\mathcal{A}$ and that in $\mathcal{A} / I$ coincide. The proof of the proposition is completed.

ExAmple. (1) If $A$ is an artin $R$-algebra then, by Lemma $1.9(2)$ and Proposition 2.9, the projectively stable category $\underline{\bmod } A$ of $\bmod A$, as well as the injectively stable category $\overline{\bmod } A$, is an Auslander-Reiten $R$-category.

(2) Let $S$ be a complete regular local commutative $k$-algebra with residue field $k$, and let $\Lambda$ be an $S$-algebra which is free of finite rank as an $S$-module. If $\Lambda$ is an isolated sigularity or nonsingular, then the projectively stable category of finitely generated $\Lambda$-modules which are free as $S$-modules is an Auslander-Reiten $k$-category; see [2], [13, (3.4.5)].

2.10. Theorem. Let $\mathcal{A}$ be a left or right Auslander-Reiten category, and let $X_{0} \rightarrow X_{1} \rightarrow \cdots \rightarrow X_{n}$ be a sectional path in $\Gamma_{\mathcal{A}}$. If there exist irreducible morphisms $f_{i}: X_{i-1} \rightarrow X_{i}$ such that $f_{1} \cdots f_{n}$ factors through some object $Y$, then one of the $X_{i}$ is a summand of $Y$.

Proof. We consider only the case where $\mathcal{A}$ is a left Auslander-Reiten category. Let $Y$ be an object in $\mathcal{A}$, and let $\mathcal{I}$ be the ideal of the morphisms factoring through summands of $Y$. By Lemma 1.9(2), $\mathcal{I}$ is 
admissible, and for an object $X$ in $\Gamma_{\mathcal{A}}$, we see that $1_{X} \in \mathcal{I}$ if and only if $X$ is isomorphic to a summand of $Y$. By Proposition $2.9, \mathcal{A} / \mathcal{I}$ is a left Auslander-Reiten category. Assume that $1_{X_{i}} \notin \mathcal{I}, i=0,1, \ldots, n$. By Proposition 2.9, $X_{0} \rightarrow X_{1} \rightarrow \cdots \rightarrow X_{n}$ is a sectional path in $\Gamma_{\mathcal{A} / I}$. If $f_{i}: X_{i-1} \rightarrow X_{i}$ are irreducible in $\mathcal{A}$ then, by Lemma 1.7(1), $\bar{f}_{i}: X_{i-1} \rightarrow X_{i}$ is irreducible in $\mathcal{A} / \mathcal{I}, i=1, \ldots, n$. By Lemma 2.7(1), $\bar{f}_{1} \cdots \bar{f}_{n} \neq \overline{0}$. That is, $f_{1} \cdots f_{n}$ does not factor through any summand of $Y$. The proof of the Theorem is completed.

As an application, we have the following interesting result concerning the extensions between indecomposable modules over an artin algebra.

2.11. Proposition. Let $A$ be an artin algebra. Assume that the Auslander-Reiten quiver of $A$ has a sectional path $X_{0} \rightarrow X_{1} \rightarrow \cdots \rightarrow$ $X_{n}$.

(1) If the $X_{i}$ are all non-projective, then $\operatorname{Ext}_{A}^{1}\left(X_{n}, \tau X_{i}\right) \neq 0$ for $0 \leq i \leq n$.

(2) If the $X_{i}$ are all non-injective, then $\operatorname{Ext}_{A}^{1}\left(X_{0}, \tau^{-} X_{i}\right) \neq 0$ for $0 \leq i \leq n$.

Proof. It suffices to prove the first statement. If none of the $X_{i}$ is projective, then $\tau X_{0} \rightarrow \tau X_{1} \rightarrow \cdots \rightarrow \tau X_{n}$ is a sectional path in the Auslander-Reiten quiver of $A$. Let $f_{j}: \tau X_{j-1} \rightarrow \tau X_{j}$ be irreducible maps in $\bmod A, j=1, \ldots, n$. By Theorem 2.10, $f_{1} \cdots f_{n}$ does not factor through any injective module in $\bmod A$. Therefore, $\operatorname{Ext}_{A}^{1}\left(X_{n}, \tau X_{i}\right) \cong D \overline{\operatorname{Hom}}_{A}\left(\tau X_{i}, \tau X_{n}\right) \neq \overline{0}$, for $i=0,1, \ldots, n$. The proof of the proposition is completed.

\section{Degrees of IRREDUCIBle MORPhisms}

Let $\mathcal{A}$ be a Krull-Schmidt category. We shall introduce the notion of degrees of irreducible morphisms in $\mathcal{A}$ and collect some of their properties. This was originally introduced and studied in a module category; see [18]. In most cases, we shall refer the details of a proof to $[18,20]$.

3.1. Definition. Let $f: X \rightarrow Y$ be an irreducible morphism in $\mathcal{A}$ with $X$ or $Y$ indecomposable. We define the left degree $d_{l}(f)$ 
of $f$ to be the minimal integer $n \geq 1$, if such an integer exists, for which there exists a morphism $\theta \in \operatorname{rad}^{n}(M, X) \backslash \operatorname{rad}^{n+1}(M, X)$ such that $\theta f \in \operatorname{rad}^{n+2}(M, Y)$; and to be infinity if such an integer does not exist. In a dual manner, we define the right degree $d_{r}(f)$ of $f$.

The following result follows immediately from the definition.

3.2. Lemma. Let $f: X \rightarrow Y$ be an irreducible morphism in $\mathcal{A}$ with $X$ or $Y$ indecomposable. If $p: Y \rightarrow Y_{1}$ is a retraction, then $d_{l}(f p) \leq d_{l}(f)$ and if $q: X_{1} \rightarrow X$ is a section, then $d_{r}(q f) \leq d_{r}(f)$.

We now state the following key observation.

3.3. Lemma. Let $X_{0} \stackrel{f_{1}}{\longrightarrow} X_{1} \longrightarrow \cdots \longrightarrow X_{n-1} \stackrel{f_{n}}{\longrightarrow} X_{n}$ be a chain of irreducible morphisms between indecomposable objects in $\mathcal{A}$. If $X_{0} \cong X_{n}$, then at least one of the $f_{i}$ has finite left degree and at least one has finite right degree.

Proof. Assume that $X_{0}=X_{n}$. Then $f_{1} \cdots f_{n} \in \operatorname{rad}\left(X_{0}, X_{0}\right)$, and hence $\left(f_{1} \cdots f_{n}\right)^{r}=0$ for some $r>0$. Setting $X_{j n+j}=X_{j}$ and $f_{j n+i}=$ $f_{i}$, for $i=1, \ldots, n ; j=1, \ldots, r-1$. Suppose that $d_{l}\left(f_{i}\right)=\infty, i=$ $1, \ldots, n$. Then $d_{l}\left(f_{i}\right)=\infty, i=1,2, \ldots, r n$. Since $f_{1} \notin \operatorname{rad}^{2}\left(X_{0}, X_{1}\right)$, it follows by induction that $f_{1} \cdots f_{i} \notin \mathrm{rad}^{i+1}\left(X_{0}, X_{i}\right), i=1,2, \ldots, r n$. In particular, $f_{1} f_{2} \cdots f_{r n} \neq 0$. This contradiction shows that one of the $f_{i}$ is of finite left degree. Dually, one of the $f_{i}$ is of finite right degree. The proof of the lemma is completed.

As an immediate consequence of Lemma 2.5, we have the following result.

3.4. Lemma. Let $f: Y \rightarrow Z$ be an irreducible morphism in $\mathcal{A}$ with $Z$ indecomposable. If $f$ is of finite left degree, then

(1) $Z$ is not pseudo-projective, and

(2) if $\mathcal{A}$ admits an Auslander-Reiten sequence $X \stackrel{\left(f^{\prime}, g^{\prime}\right)}{\longrightarrow} Y \amalg Y^{\prime} \stackrel{\left(\begin{array}{l}f \\ g\end{array}\right)}{\longrightarrow} Z$ with $Y^{\prime} \neq 0$, then $d_{l}\left(g^{\prime}\right)<d_{l}(f)$.

In the sequel, we shall collect some properties of the left degree of irreducible morphisms in a left Auslander-Reiten category. The dual 
results hold for the right degree of irreducible morphisms in a right Auslander-Reiten category.

3.5. Lemma. Let $\mathcal{A}$ be a left Auslander-Reiten category, and let $f: X \rightarrow Y$ with $Y$ indecomposable be an irreducible morphism of finite left degree in $\mathcal{A}$. If

$$
Y_{n} \rightarrow \cdots \rightarrow Y_{1} \rightarrow Y_{0}=Y
$$

is a pre-sectional path in $\Gamma_{\mathcal{A}}$ such that $\mathcal{A}$ admits an irreducible morphism from $X \amalg Y_{1}$ to $Y$, then the $Y_{i}$ are not pseudo-projective and there exist irreducible morphisms $f_{i}: \tau Y_{i-1} \rightarrow Y_{i}$ such that $d_{l}\left(f_{n}\right)<$ $\cdots<d_{l}\left(f_{1}\right)<d_{l}(f)$, and consequently, $n<d_{l}(f)$.

Proof. The result follows from a recursive application of Theorem 1.4(2), Lemmas 3.2 and 3.4. The proof of the lemma is completed.

3.6. Lemma. Let $\mathcal{A}$ be a left Auslander-Reiten category. If $X \rightarrow Y$ is an arrow in $\Gamma_{\mathcal{A}}$, then the irreducible morphisms from $X$ to $Y$ have the same left degree which is infinite in case $d_{X Y}>1$ and $d_{X Y}^{\prime}>1$.

Proof. Let $f, g: X \rightarrow Y$ be irreducible morphisms in $\mathcal{A}$. If $d_{X Y}>1$ and $d_{X Y}^{\prime}>1$, using Lemma 3.5, one deduces that $d_{l}(f)=d_{l}(g)=\infty$; for details, see $[20,(1.6)]$. Otherwise, $f=g a+h$ or $f=b g+h$, where $a \in \operatorname{Aut}(Y), b \in \operatorname{Aut}(X)$, and $h \in \operatorname{rad}^{2}(X, Y)$. It follows then from the definition that $d_{l}(f)=d_{l}(g)$. The proof of the lemma is completed.

Let $\mathcal{A}$ be a left Auslander-Reiten category. By Lemma 3.6, we may define the left degree of an arrow $X \rightarrow Y$ in $\Gamma_{\mathcal{A}}$ to be that of any irreducible morphism $f: X \rightarrow Y$. It follows from Lemma 3.3 that an oriented cycle in $\Gamma_{\mathcal{A}}$ contains at least one arrow of finite left degree.

3.7. Lemma. Let $\mathcal{A}$ be a left Auslander-Reiten category, containing an irreducible morphism $f: X \rightarrow Y_{1} \amalg Y_{2}$, where $X, Y_{1}, Y_{2}$ are indecomposable. If $d_{l}(f)<\infty$, then there exists an irreducible morphism $g: \tau Y_{1} \amalg \tau Y_{2} \rightarrow X$ such that $d_{l}(g)<d_{l}(f)$.

Proof. The result follows from Lemmas 2.5 and 3.6; for details, see $[20,(1.10)]$. The proof of the lemma is completed.

A vertex $x$ in a valued translation quiver $(\Gamma, \rho, v)$ is called left stable if $\rho^{i} x$ is defined for every $i \geq 0$, right stable if $\rho^{-i} x$ is defined for every 
$i \geq 0$, and stable if $\rho^{i} x$ is defined for every integer $i$. If $X \rightarrow Y$ is an arrow in $\Gamma_{\mathcal{A}}$ with $X, Y$ left stable, then we define the global left degree of $X \rightarrow Y$ to be the minimum of the left degrees of the arrows of the form $\tau^{i+1} Y \rightarrow \tau^{i} X$ and $\tau^{i} X \rightarrow \tau^{i} Y$ with $i \geq 0$.

3.8. Lemma. Let $\mathcal{A}$ be a left Auslander-Reiten category, and let $\Gamma_{\mathcal{A}}$ have two infinite pre-sectional paths containing only left stable objects

$$
\cdots \rightarrow X_{n} \rightarrow \cdots \rightarrow X_{1} \rightarrow X_{0}
$$

and

$$
\cdots \rightarrow Y_{n} \rightarrow \cdots \rightarrow Y_{1} \rightarrow Y_{0}
$$

with $X_{0}=Y_{0}$. If $\mathcal{A}$ has an irreducible morphism from $X_{1} \amalg Y_{1}$ to $X_{0}$, then $X_{n+1} \rightarrow X_{n}$ and $Y_{n+1} \rightarrow Y_{n}$ are of infinite global left degree for every $n \geq 0$.

Proof. Assume that $\mathcal{A}$ has an irreducible morphism from $X_{1} \amalg Y_{1}$ to $X_{0}$. Fix an integer $i \geq 0$. For each $j \geq 0$, since the $X_{n}$ are left stable, $\Gamma_{\mathcal{A}}$ has an infinite pre-sectional path

$$
\cdots \rightarrow \tau^{j} X_{n} \rightarrow \cdots \rightarrow \tau^{j} X_{i+2} \rightarrow \tau^{j} X_{i+1}
$$

such that $\mathcal{A}$ has an irreducible morphism from $\tau^{j+1} X_{i} \amalg \tau^{j} X_{i+2}$ to $\tau^{j} X_{i+1}$. By Lemma 3.5, $\tau^{j+1} X_{i} \rightarrow \tau^{j} X_{i+1}$ is of infinite left degree. Consider now the arrow $\tau^{j} X_{i+1} \rightarrow \tau^{j} X_{i}$. If $i=0$, then $\tau^{j} X_{1} \rightarrow \tau^{j} X_{0}$ is of infinite left degree since $\Gamma_{\mathcal{A}}$ has an infinite pre-sectional path

$$
\cdots \rightarrow \tau^{j} Y_{n} \rightarrow \cdots \rightarrow \tau^{j} Y_{1} \rightarrow \tau^{j} Y_{0}
$$

such that $\mathcal{A}$ has an irreducible morphism from $\tau^{j} X_{1} \amalg \tau^{j} Y_{1}$ to $\tau^{j} Y_{0}$. If $i>0$, then $\Gamma_{\mathcal{A}}$ has an infinite pre-sectional path

$$
\cdots \rightarrow \tau^{i+j} Y_{2} \rightarrow \tau^{i+j} Y_{1} \rightarrow \tau^{i+j} X_{0} \rightarrow \cdots \rightarrow \tau^{j+1} X_{i-1} \rightarrow \tau^{j} X_{i}
$$

such that $\mathcal{A}$ has an irreducible morphism from $\tau^{j} X_{i+1} \amalg \tau^{j+1} X_{i-1}$ to $\tau^{j} X_{i}$. Hence $\tau^{j} X_{i+1} \rightarrow \tau^{j} X_{i}$ is of infinite left degree. This shows that $X_{i+1} \rightarrow X_{i}$ is of infinite global left degree. By symmetry, $Y_{i+1} \rightarrow Y_{i}$ is of infinite global left degree. The proof of the lemma is completed.

3.9. Corollary. Let $\mathcal{A}$ be a left Auslander-Reiten category, and let

$$
\cdots \rightarrow X_{n} \rightarrow \cdots \rightarrow X_{1} \rightarrow X_{0} \rightarrow X_{-1} \rightarrow \cdots \rightarrow X_{-n} \rightarrow \cdots
$$


be a double infinite pre-sectional path in $\Gamma_{\mathcal{A}}$. If the $X_{n}$ are all left stable, then $X_{n+1} \rightarrow X_{n}$ is of infinite global left degree for every integer $n$.

Proof. Assume that the $X_{n}$ are all left stable. Then, for each integer $i$, there exist in $\mathcal{A}$ two infinite pre-sectional paths containing only left stable objects

$$
\cdots \rightarrow X_{n+1} \rightarrow X_{n} \rightarrow \cdots \rightarrow X_{i+1} \rightarrow X_{i}
$$

and

$$
\cdots \rightarrow \tau^{n} X_{i-n} \rightarrow \cdots \rightarrow \tau X_{i-1} \rightarrow X_{i}
$$

such that $\mathcal{A}$ has an irreducible morphism from $\tau X_{i-1} \amalg X_{i+1}$ to $X_{i}$. Now the result follows from Lemma 3.8. The proof of the corollary is completed.

3.10. Lemma. Let $\mathcal{A}$ be a left Auslander-Reiten category, and let $\Gamma_{\mathcal{A}}$ have an infinite sectional path containing only left stable objects as follows:

$$
\cdots \rightarrow X_{n} \rightarrow \cdots \rightarrow X_{1} \rightarrow X_{0} .
$$

If there exists some $i \geq 1$ such that $X_{i} \rightarrow X_{i-1}$ is of finite global left degree, then $X_{0}$ has at most two left stable immediate predecessors in $\Gamma_{\mathcal{A}}$, moreover, $X_{1} \rightarrow X_{0}$ is trivially valued in case $i>1$.

Proof. Assume that $X_{i} \rightarrow X_{i-1}$ with $i \geq 1$ be of finite global left degree. It follows from Lemma 3.8 that $d_{X_{1} X_{0}}^{\prime}=1$. We claim that the middle term of the Auslander-Reiten sequence ending with $X_{0}$ has at most two (including multiplicity) left stable indecomposable summands. Indeed, let $j \geq 0$ be such that $\tau^{j} X_{i} \rightarrow \tau^{j} X_{i-1}$ or $\tau^{j+1} X_{i-1} \rightarrow \tau^{j} X_{i}$ is of finite left degree. Note that $\Gamma_{\mathcal{A}}$ contains an infinite sectional path

$$
\cdots \rightarrow \tau^{j} X_{n} \rightarrow \cdots \rightarrow \tau^{j} X_{i+1} \rightarrow \tau^{j} X_{i} .
$$

By Lemma 3.5, $\tau^{j+1} X_{i-1} \rightarrow \tau^{j} X_{i}$ is of infinite left degree, and consequently, $\tau^{j} X_{i} \rightarrow \tau^{j} X_{i-1}$ is of finite left degree. Applying Lemma 3.5 to the sectional path $\tau^{j+i-1} X_{0} \rightarrow \tau^{j+i-2} X_{1} \rightarrow \cdots \rightarrow \tau^{j+1} X_{i-2} \rightarrow$ $\tau^{j} X_{i-1}$ (which is trivial in case $i=1$ ), we see that $\tau^{j+i-1} X_{1} \rightarrow$ $\tau^{j+i-1} X_{0}$ is of finite left degree. If the claim is false, then the middle term of the Auslander-Reiten sequence ending with $\tau^{j+i-1} X_{0}$ has a summand of the form $\tau^{j+i-1} X_{1} \amalg Y_{1} \amalg Y_{2}$, where $Y_{1}, Y_{2}$ are left stable and indecomposable. By Lemma 3.4(2), there exists an irreducible morphism $f: \tau^{j+i} X_{0} \rightarrow Y_{1} \amalg Y_{2}$ of finite left degree, and by Lemma 
3.7, we have an irreducible morphism $g: \tau Y_{1} \amalg \tau Y_{2} \rightarrow \tau^{j+i} X_{0}$ with $d_{l}(g)<d_{l}(f)$. On the other hand, $\Gamma$ contains an infinite sectional path

$$
\cdots \rightarrow \tau^{j+i} X_{n} \rightarrow \cdots \rightarrow \tau^{j+i} X_{1} \rightarrow \tau^{j+i} X_{0}
$$

such that $\mathcal{A}$ has an irreducible morphism from $\tau Y_{1} \amalg \tau Y_{2} \amalg \tau^{j+i} X_{1}$ to $\tau^{j+i} X_{0}$. By Lemma 3.5, $d_{l}(g)=\infty$. This contradiction establishes the claim. In particular, $X_{0}$ has at most two left stable immediate predecessors in $\Gamma_{\mathcal{A}}$. If $i>1$, applying the above claim to $X_{1}$, we get $d_{\tau X_{0}, X_{1}}^{\prime}=1$, that is $d_{X_{1} X_{0}}=1$. The proof of the lemma is completed.

If $P$ is a path in a valued translation quiver $(\Gamma, \rho, v)$ containing only left stable vertices then, for each $i \geq 0$, we denote by $\rho^{i} P$ the path in $\Gamma$ obtained by applying $\rho^{i}$ to $P$.

3.11. Lemma. Let $\mathcal{A}$ be a left Auslander-Reiten category, and let $\Gamma_{\mathcal{A}}$ have an infinite sectional path containing only stable objects

$$
P: \quad \cdots \rightarrow X_{n} \rightarrow \cdots \rightarrow X_{1} \rightarrow X_{0} .
$$

If $P$ contains infinitely many arrows of finite global left degree, then so does $\tau^{m} P$ for every $m \geq 0$.

Proof. Assume that $P$ contains infinitely many arrows of finite global left degree. Fix a positive integer $m$. To show that $\tau^{m} P$ contains infinitely many arrows of finite global left degree, it suffices to show that if $X_{s+1} \rightarrow X_{s}$ is of finite global left degree for some $s>m$, then there exists some $i$ with $s-m \leq i \leq s$ such that $\tau^{m} X_{i+1} \rightarrow \tau^{m} X_{i}$ is of finite global left degree. Indeed, let $r \geq 0$ be such that $\tau^{r} X_{s+1} \rightarrow \tau^{r} X_{s}$ or $\tau^{r+1} X_{s} \rightarrow \tau^{r} X_{s+1}$ is of finite left degree. Applying Lemma 3.5 to the infinite sectional path

$$
\cdots \rightarrow \tau^{r} X_{n+1} \rightarrow \tau^{r} X_{n} \rightarrow \cdots \rightarrow \tau^{r} X_{s+2} \rightarrow \tau^{r} X_{s+1},
$$

we see that it is $\tau^{r} X_{s+1} \rightarrow \tau^{r} X_{s}$ which has finite left degree. If $r \geq$ $m$, then $\tau^{m} X_{s+1} \rightarrow \tau^{m} X_{s}$ is of finite global left degree. Otherwise, applying Lemma 3.5 to the sectional path

$$
\tau^{m} X_{s-m+r} \rightarrow \tau^{m} X_{s-m+r+1} \rightarrow \cdots \rightarrow \tau^{r+1} X_{s-1} \rightarrow \tau^{r} X_{s},
$$

we see that $\tau^{m} X_{s-m+r+1} \rightarrow \tau^{m} X_{s-m+r}$ is of finite left degree with $s-m \leq s-m+r<s$. The proof of the lemma is completed. 


\section{Semi-Stable VAlued TRAnslation Quivers}

The objective of this section is to describe semi-stable valued translation quivers. Let $(\Gamma, \rho, v)$ be a valued translation quiver. We say that $(\Gamma, \rho, v)$ is left stable (respectively, right stable, stable, $\rho$-periodic) if each vertex in $\Gamma$ is left stable (respectively, right stable, stable, $\rho$ periodic). Given a valued quiver $(\Delta, v)$ with no oriented cycle, one defines a stable valued translation quiver $\mathbb{Z} \Delta$ as follows. The vertices are pairs $(n, x)$, where $n \in \mathbb{Z}$ and $x \in \Delta_{0}$; each arrow $x \rightarrow y$ with valuation $\left(v_{x y}, v_{x y}^{\prime}\right)$ in $\Delta$ induces, for each integer $n$, two arrows $(n, x) \rightarrow(n, y)$ and $(n+1, y) \rightarrow(n, x)$ in $\mathbb{Z} \Delta$ with valuations $\left(v_{x y}, v_{x y}^{\prime}\right)$ and $\left(v_{x y}^{\prime}, v_{x y}\right)$, respectively; and the translation is defined by sending $(n, x)$ to $(n+1, x)$, for all $n \in \mathbb{Z}$ and $x \in \Delta_{0}$. Next, we recall the definition of the orbit graph $\mathcal{O}(\Gamma)$ of $\Gamma$. For each vertex $x$ in $\Gamma$, the $\rho$-orbit $o(x)$ of $x$ is the set of vertices of the form $\rho^{n} x$ with $n \in \mathbb{Z}$. Now the vertices in $\mathcal{O}(\Gamma)$ are the $\rho$-orbits in $\Gamma$, and $\mathcal{O}(\Gamma)$ contains an edge $o_{1}-o_{2}$ if and only if $\Gamma$ contains an arrow $x_{1} \rightarrow x_{2}$ or $x_{2} \rightarrow x_{1}$ with $x_{i} \in o_{i}, i=1,2$. Note that $\mathcal{O}(\Gamma)$ is not necessarily locally finite even if $\Gamma$ is locally finite. Furthermore, a valued translation quiver morphism $\varphi:(\Sigma, \sigma, u) \rightarrow(\Gamma, \rho, v)$ is a quiver morphism $\varphi: \Sigma \rightarrow \Gamma$ such that $\varphi(\Sigma)$ is a valued translation subquiver of $\Gamma$. Such a morphism is called an embedding if its action on the vertices is injective, and an embedding $\varphi:(\Sigma, \sigma, u) \rightarrow(\Gamma, \rho, v)$ is called orbit-complete if $\varphi(\Sigma)$ meets each $\rho$-orbit in $\Gamma$. Finally, a full connected valued subquiver $\Delta$ of $\Gamma$ is called a section if $\Delta$ is convex, contains no oriented cycle, and meets exactly once each $\rho$-orbit in $\Gamma$. In this case, there exists an orbit-complete embedding $\Gamma \rightarrow \mathbb{Z} \Delta$ sending $\rho^{n} x$ to $(n, x)$, which we call the canonical embedding; see [20, (2.1),(2.3)].

4.1. Lemma. Let $(\Gamma, \rho, v)$ be a valued translation quiver which is connected and left stable.

(1) If $x, y \in \Gamma_{0}$, then there exists $r \geq 0$ such that $\rho^{r} x$ is a predecessor of $y$.

(2) If $\Gamma$ contains a non-trivial path from $x$ to $y$, then $x=\rho^{n} y$ with $n>0$ or $\Gamma$ contains a sectional path $x=x_{0} \rightarrow \cdots \rightarrow x_{s} \rightarrow x_{s+1}=\rho^{r} y$ with $r, s \geq 0$. 
Proof. (1) Being connected, $\Gamma$ contains a walk $y=x_{0}-x_{1}-\cdots$ $-x_{t}=x$. Since $\rho$ is defined everywhere, an easy induction on $t$ shows that $\Gamma$ contains a path $\rho^{r} x=y_{s} \rightarrow \cdots \rightarrow y_{0}=y$ with $r, s \geq 0$.

(2) Let $x=y_{0} \rightarrow \cdots \rightarrow y_{t-1} \rightarrow y_{t}=y$ with $t>0$ be a path in $\Gamma$. If $t=1$, then $x \rightarrow y$ is a sectional path. Assume that $t>1$ and that either $x=\rho^{n} y_{t-1}$ for some $n>0$ or there exists a sectional path $x=z_{0} \rightarrow \cdots \rightarrow z_{s} \rightarrow z_{s+1}=\rho^{r} x$ with $s, r \geq 0$. In the first case, $x \rightarrow \rho^{n} y$ is a sectional path. In the second case, $\Gamma$ contains a path $x=z_{0} \rightarrow \cdots \rightarrow z_{s} \rightarrow z_{s+1} \rightarrow \rho^{r} y$. If this path is not sectional, then $z_{s}=\rho^{r+1} y$. If $s=0$, then $x=\rho^{r+1} y$ with $r+1>0$. Otherwise, $x=z_{0} \rightarrow \cdots \rightarrow z_{s-1} \rightarrow z_{s}=\rho^{r} y$ is a sectional path. The proof of the lemma is completed.

The following result describes the left stable valued translations with no oriented cycle. This is a generalization of Theorem 3.4 stated in [19], where the condition that $\Gamma$ contain at most finitely many injective vertices is imposed.

4.2. TheOREM. Let $(\Gamma, \rho, v)$ be a connected left stable valued translation quiver. If $\Gamma$ contains no oriented cycle, then there exists an orbit-complete embedding $\Gamma \rightarrow \mathbb{Z} \Delta$, where $\Delta$ is a valued quiver with a unique sink but no oriented cycle, and if in addition the paths in $\Gamma$ with injective end-points have bounded number of hooks, then $\Delta$ can be chosen to be a section in $\Gamma$.

Proof. Assume that $\Gamma$ contains no oriented cycle. We shall first construct the valued quiver $\Delta$ from the orbit graph $\mathcal{O}(\Gamma)$ with the canonical orientation and valuation. For this purpose, fix arbitrarily a vertex $a$ in $\Gamma$. For $n \geq 0$, let $\Delta^{(n)}$ be the full valued subquiver of $\Gamma$ generated by the vertices $x$ for which there exists some $r \geq 0$ such that $\rho^{r} x$ is a predecessor of $\rho^{r+n} a$ while there exists no $s \geq 0$ such that $\rho^{s} x$ is a predecessor of $\rho^{s+n+1} a$. Since $\Gamma$ contains no oriented cycle, $\rho^{n} a \in \Delta^{(n)}$ for every $n \geq 0$. Now we divide the proof into several sub-lemmas.

(1) For each $n \geq 0, \Delta^{(n)}$ is convex in $\Gamma$. Let $x, z$ be vertices in $\Delta^{(n)}$, and $y$ a vertex lying on a path from $x$ to $z$. If $r \geq 0$ is such that $\rho^{r} z$ is a predecessor of $\rho^{r+n} a$, then $\rho^{r} y$ is a predecessor of $\rho^{r+n} a$. If there exists some $s \geq 0$ such that $\rho^{s} y$ is a predecessor of $\rho^{s+n+1} a$, then $\rho^{s} x$ is a predecessor of $\rho^{s+n+1} a$, a contradiction. Thus $y \in \Delta^{(n)}$. 
(2) If $x \in \Delta^{(n)}$ for some $n \geq 0$, then $\rho^{m} x \notin \Delta^{(n)}$ for every $m \neq 0$. Let $r \geq 0$ be such that $\rho^{r} x$ is a predecessor of $\rho^{r+n} a$. If $m>0$, then $\rho^{r}\left(\rho^{m} x\right)$ is a predecessor of $\rho^{r+n+m} a$, and hence a predecessor of $\rho^{r+n+1} a$. Therefore, $\rho^{m} x \notin \Delta^{(n)}$. Since $x \in \Delta^{(n)}$, this in turn implies that $\rho^{m} x \notin \Delta^{(n)}$ for $m<0$.

(3) If $x \in \Delta^{(n)}$ for some $n \geq 0$, then $\rho x \in \Delta^{(n+1)}$, and $\rho^{-} x \in$ $\Delta^{(n-1)}$ whenever $n>0$ and $x$ is non-injective. Let $r \geq 0$ be such that $\rho^{r} x$ is a predecessor of $\rho^{r+n} a$. Then $\rho^{r}(\rho x)$ is a predecessor of $\rho^{r+(n+1)} a$. If $s \geq 0$, then $\rho^{s}(\rho x)=\rho^{s+1} x$ is not a predecessor of $\rho^{(s+1)+n+1} a=\rho^{s+(n+1)+1} a$. Thus $\rho x \in \Delta^{(n+1)}$. Suppose now that $n>0$ and $x$ is not injective. Then $\rho^{r+1}\left(\rho^{-} x\right)=\rho^{r} x$ is a predecessor of $\rho^{r+n} a=\rho^{(r+1)+(n-1)} a$. Moreover, if there exists some $s \geq 0$ such that $\rho^{s}\left(\rho^{-} x\right)$ is a predecessor of $\rho^{s+(n-1)+1} a=\rho^{s+n} a$, then $\rho^{s} x$ is a predecessor of $\rho^{s+n+1} a$, a contradiction. Thus $\rho^{-} x \in \Delta^{(n-1)}$.

(4) If $x \rightarrow y$ is an arrow with $y \in \Delta^{(n)}$, then $x$ lies in $\Delta^{(n)}$ or $\Delta^{(n+1)}$. Let $r \geq 0$ be such that $\rho^{r} y$ is a predecessor of $\rho^{r+n} a$. Then $\rho^{r} x$ is a predecessor of $\rho^{r+n} a$. Suppose that $x \notin \Delta^{(n)}$. Then $\rho^{s} x$ is a predecessor of $\rho^{s+n+1} a=\rho^{s+(n+1)} a$ for some $s \geq 0$. If $\rho^{t} x$ is a predecessor of $\rho^{t+(n+1)+1} a$ for some $t \geq 0$, then $\rho^{t+1} y$ is a predecessor of $\rho^{(t+1)+n+1} a$, a contradiction. Hence $x \in \Delta^{(n+1)}$.

(5) For each vertex $x$ in $\Gamma$, there exist some $s, n \geq 0$ such that $\rho^{s} x \in \Delta^{(n)}$. Moreover, $\ell(x)=n-s$ is well-defined such that $\ell(\rho x)=$ $\ell(x)+1$. By Lemma 4.1(1), there exists some $s \geq 0$ such that $\rho^{s} x$ is a predecessor of $a$. Since $a \in \Delta^{(0)}$, we deduce from (4) that $\rho^{s} x \in \Delta^{(n)}$ for some $n \geq 0$. Furthermore, let $r, m \geq 0$ be such that $\rho^{r} x \in \Delta^{(m)}$. We may assume that $m \geq n$. By (3), $\rho^{m-n+s} x \in \Delta^{(m)}$, and by (2), $r=m-n+s$. That is $n-s=m-r$. Thus $\ell(x)$ is well-defined. Finally, by $(3), \rho^{s}(\rho x)=\rho\left(\rho^{s} x\right) \in \Delta^{(n+1)}$. Hence $\ell(\rho x)=(n+1)-s=$ $(n-s)+1=\ell(x)+1$.

(6) Let $x \rightarrow y$ be an arrow in $\Gamma$. If $r, s \geq 0$ are such that $\rho^{r} x, \rho^{s} y \in$ $\Delta^{(n)}$ for some $n \geq 0$, then $r=s$ or $r=s-1$. By (4), $\rho^{s} x$ lies in $\Delta^{(n)}$ or $\Delta^{(n+1)}$. On the other hand, by $(3), \rho^{r+1} x \in \Delta^{(n+1)}$. By $(2), r=s$ or $r+1=s$. 
(7) If $o_{1}-o_{2}$ is an edge in $\mathcal{O}(\Gamma)$, then $o_{1} \neq o_{2}$, and some $\Delta^{(n)}$ with $n \geq 0$ contains an arrow $x \rightarrow y$ or $y \rightarrow x$ with $x \in o_{1}$ and $y \in o_{2}$. By the definition of $\mathcal{O}(\Gamma)$, we may assume that $\Gamma$ contains an arrow $x \rightarrow y$ with $x \in o_{1}$ and $y \in o_{2}$. Suppose that $o_{1}=o_{2}$. Then $y=\rho^{r} x$ for some integer $r$. Since $\Gamma$ contains no oriented cycle, we have $r<0$. Thus $x$ is a predecessor of $\rho^{r+1} x$. This yields an oriented cycle from $\rho^{r+1} x$ from $\rho^{r+1} x$ passing through $x$, a contradiction. Therefore, $o_{1} \neq o_{2}$. Furthermore, by (3) and (5), there exist $r, s, n \geq 0$ such that $\rho^{r} x, \rho^{s} y \in \Delta^{(n)}$. By (6), either $r=s$ or $r=s-1$. Note that $\rho^{r} x \rightarrow \rho^{r} y$ and $\rho^{r+1} y \rightarrow \rho^{r} x$ are arrows in $\Gamma$. Thus either $\rho^{r} x \rightarrow \rho^{s} y$ or $\rho^{s} y \rightarrow \rho^{r} x$ is an arrow in $\Delta^{(n)}$.

Now, let $o_{1}-o_{2}$ be an edge in $\mathcal{O}(\Gamma)$. By $(7)$, there exists some $n \geq 0$ such that $\Delta^{(n)}$ contains an arrow $x \rightarrow y$ or $y \rightarrow x$ with $x \in o_{1}$ and $y \in o_{2}$. We orient the edge $o_{1}-o_{2}$ from $o_{1}$ to $o_{2}$ with valuation $\left(v_{x y}, v_{x y}^{\prime}\right)$ in the first case, and from $o_{2}$ to $o_{1}$ with valuation $\left(v_{y x}, v_{y x}^{\prime}\right)$ in the second case. It follows from (3) and (2) that this orientation and the valuation do not depend on the choice of $n$. Hence, we have a well-defined orientation and valuation for $\mathcal{O}(\Gamma)$, and we denote by $\Delta$ the resulted valued quiver.

(8) $\Delta$ contains no oriented cycle and has o(a) as an unique sink. Assume that $o_{1} \rightarrow \cdots \rightarrow o_{t}$ is a path in $\Delta$. It follows from (3) and (5) that there exists some $n \geq 0$ such that $\Delta^{(n)}$ contains vertices $x_{i} \in o_{i}$, $i=1, \ldots, t$. By the definition of the orientation on $\Delta$, there exists a path $x_{1} \rightarrow x_{2} \rightarrow \cdots \rightarrow x_{t}$ in $\Delta^{(n)}$. In particular, $\Delta$ contains no oriented cycle. Now let $o=o(y)$ be a vertex in $\Delta$. By (5), there exist $s, n \geq 0$ such that $\rho^{s} y \in \Delta^{(n)}$. Thus there exists some $r \geq 0$ such that $\Gamma$ contains a path $\rho^{r+s} y=y_{0} \rightarrow y_{1} \rightarrow \cdots \rightarrow y_{m}=\rho^{r+n} a$. By (3), $\rho^{r+s} y, \rho^{r+n} a \in \Delta^{(r+n)}$. By (1), the preceding path lies entirely in $\Delta^{(r+n)}$. This gives rise to a path $o(y) \rightarrow o\left(y_{1}\right) \rightarrow \cdots \rightarrow o(a)$ in $\Delta$. Hence $o(a)$ is the unique sink in $\Delta$.

(9) There exists an orbit-complete embedding $\varphi: \Gamma \rightarrow \mathbb{Z} \Delta$. For $x \in$ $\Gamma_{0}$, we define $\varphi_{0}(x)=(\ell(x), o(x))$. By $(5), \varphi_{0}(\rho x)=(\ell(\rho x), o(\rho x))=$ $(\ell(x)+1, o(x))$. Thus $\varphi_{0}$ is compatible with the translations. Let $x, y$ be vertices in $\Gamma$. We claim that $\Gamma$ contains an arrow $\alpha: x \rightarrow y$ if and only if $\mathbb{Z} \Delta$ contains an arrow $\alpha^{\prime}:(\ell(x), o(x)) \rightarrow(\ell(y), o(y))$ and we define $\varphi_{1}(\alpha)=\alpha^{\prime}$. First, assume that $\alpha^{\prime}$ exists. By construction, $\Delta$ contains either an arrow $o(x) \rightarrow o(y)$ with $\ell(x)=\ell(y)$ or an arrow 
$o(y) \rightarrow o(x)$ with $\ell(x)=\ell(y)+1$. By the definition of the orientation on $\Delta$, there exists $t \geq 0$ such that $\Delta^{(t)}$ contains an arrow $\rho^{p} x \rightarrow \rho^{q} y$ with $\ell(x)=\ell(y)$ or an arrow $\rho^{q} y \rightarrow \rho^{p} x$ with $\ell(x)=\ell(y)+1$ where $p, q$ are integers. If the first situation occurs, then $t-p=\ell(x)=\ell(y)=$ $t-q$ that is $p=q$, and hence $x \rightarrow y$ is an arrow in $\Gamma$. If the second situation occurs, then $t-p=\ell(x)=\ell(y)+1=t-q+1$ that is $q=p+1$, and consequently, $x \rightarrow y$ is also an arrow in $\Gamma$. Conversely, assume that $\alpha$ exists. Let $s, n \geq 0$ such that $\rho^{s} y \in \Delta^{(n)}$. Since $\rho^{s} x \rightarrow \rho^{s} y$ is an arrow in $\Gamma$, by $(4), \rho^{s} x$ lies in $\Delta^{(n)}$ or $\Delta^{(n+1)}$. In the first case, $\Delta$ contains an arrow $o(x) \rightarrow o(y)$ with $\ell(x)=\ell(y)$, and hence $\alpha^{\prime}$ exists. In the second case, $\ell(x)=(n+1)-s=(n-s)+1=\ell(y)+1$. By (3), $\rho^{s+1} y \in \Delta^{(n+1)}$, and hence $\Delta^{(n+1)}$ contains an arrow $\rho^{s+1} y \rightarrow \rho^{s} x$. By definition, $o(y) \rightarrow o(x)$ is an arrow in $\Delta$, and hence $\mathbb{Z} \Delta$ has an arrow $(\ell(y)+1, o(x)) \rightarrow(\ell(y), o(y))$, that is, $\alpha^{\prime}$ exists. This establishes our claim, and yields a valued translation quiver morphism $\varphi=\left(\varphi_{0}, \varphi_{1}\right)$ from $\Gamma$ to $\mathbb{Z} \Delta$ such that $\varphi(\Gamma)$ is a valued translation subquiver of $\mathbb{Z} \Delta$ which meets every translation orbit. Finally, let $x, y$ be vertices in $\Gamma$ such that $(\ell(x), o(x))=(\ell(y), o(y))$. We may assume that $y=\rho^{m} x$ with $m \geq 0$ and $\rho^{s} y \in \Delta^{(n)}$ with $s, n \geq 0$. Then $\rho^{m+s} x \in \Delta^{(n)}$. Hence $n-(m+s)=\ell(x)=\ell(y)=n-s$, and consequently $m=0$. This shows that $\varphi$ is an orbit-complete embedding.

To conclude the proof, assume that the paths in $\Gamma$ with injective end-points have bounded number of hooks. We shall show that there exists some $t \geq 0$ such that $\Delta^{(n)}$ with $n \geq t$ contains no injective vertex. Suppose that this is not the case. Let $q_{0}$ be an injective vertex in $\Delta^{\left(n_{0}\right)}$ with $n_{0} \geq 0$. Since $\Gamma$ is connected and contains no projective vertex, there exists some $m_{0}>n_{0}$ such that $\Gamma$ contains a non-sectional path from $\rho^{m_{0}} a$ to $q_{0}$. Now there exists some $n_{1}>m_{0}$ such that $\Delta^{\left(n_{1}\right)}$ contains an injective vertex $q_{1}^{\prime}$. Let $r \geq 0$ be minimal such that $\Gamma$ contains a path $\zeta$ from $\rho^{r} q_{1}^{\prime}$ to $\rho^{r+n_{1}} a$. By the minimality of $r$, the path $\zeta$ contains an injective vertex $q_{1}$. Therefore, $\Gamma$ contains a non-sectional path from $q_{1}$ to $q_{0}$. Repeating this argument, $\Gamma$ contains paths with injective end-points which have arbitrarily many hooks, a contradiction.

Let $o$ be an $\rho$-orbit in $\Gamma$. By (5) and (3), there exists some $n \geq$ $t$ such that $\Delta^{(n)}$ contains a vertex $z$ lying in $o$. If $n>t$, by (3), $\rho^{-} z \in \Delta^{(n-1)}$. Using induction, we see that $\rho^{t-n} z \in \Delta^{(t)}$. Thus 
$\Delta^{(t)}$ meets every $\rho$-orbit in $\Gamma$. Furthermore, let $x$ be a vertex in $\Delta^{(t)}$. Let $r \geq 0$ be minimal such that $\rho^{r} x$ is a predecessor of $\rho^{r+t} a$. Since $\rho^{r} x, \rho^{r+n} a \in \Delta^{(r+n)}$ with $r+t \geq t$, any path from $\rho^{r} x$ to $\rho^{r+t} a$ contains no injective vertex. Thus $r=0$ by the minimality of $r$. This shows that $\Delta^{(t)}$ is connected and has $\rho^{t} a$ as an unique sink. Since $\Gamma$ contains no oriented cycle, by (1) and (2), $\Delta^{(t)}$ is a section in $\Gamma$. Now the canonical embedding $\Gamma \rightarrow \mathbb{Z} \Delta^{(t)}$ is a desired embedding. The proof of the theorem is completed.

A valued translation quiver is called smooth if it is trivially valued and each vertex has at most two immediate predecessors and at most two immediate successors.

4.3. Lemma. Let $(\Gamma, \rho, v)$ be a connected left stable valued translation quiver which is smooth and contains a path $\rho^{r} x_{1} \rightarrow x_{s} \rightarrow \cdots \rightarrow x_{1}$ with $r>s$. If the $\rho$-orbits of $x_{1}, \ldots, x_{s}$ are pairwise different and not $\rho$-periodic, then they are the only $\rho$-orbits in $\Gamma$.

Proof. Assume that the $\rho$-orbits $o\left(x_{1}\right), \ldots, o\left(x_{s}\right)$ are pairwise different and not $\rho$-periodic. Applying repeatedly $\rho^{r}$, we get an infinite path

$$
\cdots \rightarrow \rho^{2 r} x_{1} \rightarrow \rho^{r} x_{s} \rightarrow \cdots \rightarrow \rho^{r} x_{1} \rightarrow x_{s} \rightarrow \cdots \rightarrow x_{1}
$$

in $\Gamma$, which is sectional since the $x_{i}$ are not $\rho$-periodic. Let $x$ be a vertex in $\Gamma$. By Lemma 4.1(1), there exists some $t \geq 0$ such that $\rho^{t} x$ is an predecessor of $x_{1}$. It suffices to prove the following claim: if $\Gamma$ contains a path of length $n \geq 0$ from a vertex $y$ to $x_{1}$, then $y$ lies in one of $o\left(x_{1}\right), \ldots, o\left(x_{s}\right)$. This is trivially true if $n=0$. Assume that $n>0$ and the claim holds for $n-1$. Then $y$ is an immediate predecessor of some $\rho^{j} x_{i}$, where $1 \leq i \leq s$ and $j$ is an integer. If $j<0$, then $\rho^{-j} y$ is an immediate predecessor of $x_{i}$. Thus we may assume with no loss of generality that $j \geq 0$. Suppose first that $i=1$. Let $m \geq 0$ be such that $j+m=r q$ with $q \geq 1$. Then $\rho^{m} y$ is an immediate predecessor of $\rho^{r q} x_{1}$. If $s>1$ then, since $\Gamma$ is smooth, $\rho^{m} y=\rho^{r(q-1)+1} x_{s}$ or $\rho^{m} y=\rho^{r q} x_{2}$. If $s=1$, then $\rho^{m} y=\rho^{r(q+1)} x_{1}$ or $\rho^{m} y=\rho^{r(q-1)+1} x_{1}$. Therefore, $y$ lies in one of $o\left(x_{1}\right), \ldots, o\left(x_{s}\right)$. Suppose now that $1<i \leq s$. Since $\Gamma$ is left stable, there exists another infinite sectional path

$$
\cdots \rightarrow \rho^{2 r+j} x_{1} \rightarrow \rho^{r+j} x_{s} \rightarrow \cdots \rightarrow \rho^{r+j} x_{1} \rightarrow \rho^{j} x_{s} \rightarrow \cdots \rightarrow \rho^{j} x_{1}
$$


in $\Gamma$. If $i<s$, then $y=\rho^{j} x_{i+1}$ or $y=\rho^{j+1} x_{i-1}$. If $i=s$, then $y=\rho^{j+r} x_{1}$ or $y=\rho^{j+1} x_{s-1}$. This proves our claim. The proof of the lemma is completed.

Next we shall deal with semi-stable valued translation quivers containing oriented cycles. Recall that a vertex $x$ in $\Gamma$ is a coray vertex if $\Gamma$ has an infinite sectional path, called a coray,

$$
\cdots \rightarrow x_{n} \rightarrow x_{n-1} \rightarrow \cdots \rightarrow x_{2} \rightarrow x_{1}=x,
$$

with pairwise different vertices and trivially valued arrows such that, for each $i>1$, the path $x_{i+1} \rightarrow x_{i} \rightarrow \cdots \rightarrow x_{2} \rightarrow x_{1}$ is the only sectional path of length $i$ in $\Gamma$ ending with $x$. Let this be the case. For each positive integer $n$, we construct a valued translation quiver $\Gamma[x, n]$ as follows. The vertices of $\Gamma[x, n]$ are those of $\Gamma$ plus the new vertices $(i, j)$, where $i \geq 1$ and $1 \leq j \leq n$. The arrows of $\Gamma[x, n]$ are the arrows in $\Gamma$ with the same valuation which are different from the arrows $y \rightarrow x_{i}$ with $y \neq x_{i+1}$ and $i \geq 1$, plus the following new arrows with trivial valuation:

(1) $(i+1, j) \rightarrow(i, j)$, where $i \geq 1$ and $1 \leq j \leq n$;

(2) $(i, j+1) \rightarrow(i+1, j)$, where $i \geq 1$ and $1 \leq j<n$;

(3) $(n+i-1,1) \rightarrow x_{i}$, where $i \geq 1$;

(4) $y \rightarrow(i, n)$, where $i \geq 1$ and $y \rightarrow x_{i}$ is an arrow in $\Gamma$ with $y \neq x_{i+1}$.

The translation $\sigma$ of $\Gamma[x, n]$ is defined so that, for $z \in \Gamma_{0}$, we have $\sigma z=\rho z$ in case $\rho z$ is defined and $z \neq x_{i}$ for every $i \geq 1$, and $\sigma z=(n+i, 1)$ in case $z=x_{i}$ for some $i \geq 1$, and $\sigma(i, j)=(i, j+1)$ for $i \geq 1$ and $1 \leq j<n$, and finally, $\sigma(i, n)=\rho x_{i}$ whenever $\rho x_{i}$ is defined. We say that $\Gamma[x, n]$ is obtained from $\Gamma$ by inserting $n$ corays at $x$. Note that if $\Gamma$ is left stable, then $\Gamma[x, n]$ is left stable and contains $n$ new injective vertices $(1,1), \ldots,(n, 1)$. Dually, we have the notions of a ray vertex and a ray, and construct a valued translation quiver $[x, n] \Gamma$ from $\Gamma$ by inserting $n$ rays at a ray vertex $x$. If $\Gamma$ is right stable, then $[x, n] \Gamma$ is right stable and contains $n$ new projective vertices.

One calls $\Gamma$ a stable tube if $\Gamma \cong \mathbb{Z} A_{\infty} /\left\langle\sigma^{n}\right\rangle$, where $\sigma$ is the translation of $\mathbb{Z} A_{\infty}$ and $n$ is a positive integer called the rank of $\Gamma$. We shall say that $\Gamma$ is a coray tube (respectively, ray tube) if it is 
obtained from a stable tube by a finite number of successive corayinsertions (respectively, ray-insertions). The ray tubes and co-ray tubes do appear as Auslander-Reiten components of artin algebras; see [24]. They can be characterized by the following result and its dual.

4.4. TheOREM. A connected valued translation quiver $(\Gamma, \rho, v)$ is a coray tube if and only if it is left stable, smooth, and contains a path

$$
\rho^{r+m_{1}} q_{1} \rightarrow \rho^{m_{s}} q_{s} \rightarrow \cdots \rightarrow \rho^{m_{2}} q_{2} \rightarrow \rho^{m_{1}} q_{1},
$$

where $1 \leq s<r$, and $0 \leq m_{1} \leq m_{2} \leq \cdots \leq m_{s} \leq r+m_{1}$, and $q_{1}, \ldots, q_{s}$ are pairwise distinct injective vertices.

Proof. For the necessity, let $\Gamma^{(0)}, \Gamma^{(1)}, \ldots, \Gamma^{(m)}=\Gamma$ be valued translations quivers, where $\Gamma^{(0)}$ is a stable tube of rank $n$ while $\Gamma^{(k)}$ with $k>0$ is obtained from $\Gamma^{(k-1)}$ by inserting $s_{k}(\geq 1)$ corays. By construction, the $\Gamma^{(k)}$ are left stable and smooth. We shall prove by induction that $\Gamma^{(k)}$ with $k>0$ contains a desired path. Indeed, the injective vertices in $\Gamma^{(1)}$ are $q_{j 1}=(j, 1), j=1, \ldots, s_{1}$, and $\Gamma^{(1)}$ contains a path $\rho^{n+s_{1}} q_{11} \rightarrow q_{s_{1}, 1} \rightarrow q_{s_{1}-1,1} \rightarrow \cdots \rightarrow q_{11}$ with $n+s_{1}>$ $s_{1} \geq 1$. Suppose that $\Gamma^{(k)}$ with $k \geq 1$ contains a path

$$
\begin{aligned}
\rho^{r} q_{11} & \rightarrow \rho^{n_{k}} q_{s_{k}, k} \rightarrow \cdots \rightarrow \rho^{n_{k}} q_{1, k} \rightarrow \rho^{n_{k-1}} q_{s_{k-1}, k-1} \\
\rightarrow \rho^{n_{2}} q_{12} & \rightarrow q_{s_{1}, 1} \rightarrow \cdots \rightarrow q_{11},
\end{aligned}
$$

where $0<n_{2}<\cdots<n_{k} \leq r$, and $s_{1}+\cdots+s_{k}<r$, and the $q_{j i}$ with $1 \leq$ $i \leq k$ and $1 \leq j \leq s_{i}$ are the injective vertices in $\Gamma^{(k)}$. Then the corayvertices in $\Gamma^{(k)}$ are the $\rho^{l} q_{1,1}$ with $0 \leq l \leq r-n_{k}-2$ and the $\rho^{l} q_{1 i}$ with $1<i \leq k$ and $0 \leq l \leq n_{i}-n_{i-1}-2$. We consider only the case where $\Gamma^{(k+1)}=\Gamma^{(k)}\left[\rho^{l-1} q_{1 i}, s_{k+1}\right]$ with $1<i \leq k$ and $1 \leq l \leq n_{i}-n_{i-1}-1$, since the case where $i=1$ is similar. Let $q_{1, k+1}, \ldots, q_{s_{k+1}, k+1}$ be the new injective vertices of $\Gamma^{(k+1)}$. By construction, $\Gamma^{(k+1)}$ contains paths $\rho^{l} q_{s_{i}, i} \rightarrow \cdots \rightarrow \rho^{l} q_{1, i} \rightarrow q_{s_{k+1}, k+1} \rightarrow \cdots \rightarrow q_{1, k+1}$ and

$$
\begin{aligned}
\rho^{s_{k+1}+n_{i}-n_{i-1}-l} q_{s_{k+1}, k+1} \rightarrow \cdots & \rightarrow \rho^{s_{k+1}+n_{i}-n_{i-1}-l} q_{1, k+1} \rightarrow \\
& \rightarrow q_{s_{i-1}, i-1} \rightarrow \cdots \rightarrow q_{1, i-1}
\end{aligned}
$$


Thus $\Gamma^{(k+1)}$ contains a desired path

$$
\begin{aligned}
\rho^{r+s_{k+1}} q_{11} & \rightarrow \rho^{s_{k+1}+n_{k}} q_{s_{k}, k} \rightarrow \cdots \rightarrow \rho^{s_{k+1}+n_{k}} q_{1, k} \rightarrow \cdots \\
& \rightarrow \rho^{s_{k+1}+n_{i}} q_{s_{i}, i} \rightarrow \cdots \rightarrow \rho^{s_{k+1}+n_{i}} q_{1, i} \\
& \rightarrow \rho^{s_{k+1}+n_{i}-l} q_{s_{k+1}, k+1} \rightarrow \cdots \rightarrow \rho^{s_{k+1}+n_{i}-l} q_{1, k+1} \\
& \rightarrow \rho^{n_{i-1}} q_{s_{i-1}, i-1} \rightarrow \cdots \rightarrow \rho^{n_{i-1}} q_{1, i-1} \rightarrow \cdots \\
& \rightarrow q_{s_{1}, 1} \rightarrow \cdots \rightarrow q_{11} .
\end{aligned}
$$

Conversely, assume that $\Gamma$ is left stable, smooth and contains a path

$$
(*): \quad \rho^{r} x_{1} \rightarrow x_{s} \rightarrow \cdots \rightarrow x_{1}
$$

as stated in the theorem. Applying repeatedly $\rho^{r}$ to this path, we get an infinite sectional path

$$
P: \quad \cdots \rightarrow \rho^{2 r} x_{1} \rightarrow \rho^{r} x_{s} \rightarrow \cdots \rightarrow \rho^{r} x_{1} \rightarrow x_{s} \rightarrow \cdots \rightarrow x_{1}
$$

in $\Gamma$. Rewrite the path $(*)$ in the following form:

$$
\begin{gathered}
(* *) \quad \rho^{r+n_{1}} q_{11} \rightarrow \rho^{n_{k}} q_{s_{k}, k} \rightarrow \cdots \rightarrow \rho^{n_{k}} q_{1, k} \rightarrow \rho^{n_{k-1}} q_{s_{k-1}, k-1} \rightarrow \cdots \\
\rightarrow \rho^{n_{2}} q_{12} \rightarrow \rho^{n_{1}} q_{s_{1}, 1} \rightarrow \cdots \rightarrow \rho^{n_{1}} q_{11},
\end{gathered}
$$

where $0 \leq n_{1}<n_{2}<\cdots<n_{k} \leq r+n_{1}$, and $s_{1}+\cdots+s_{k}=s<r$, and the $q_{j i}$ with $1 \leq i \leq k$ and $1 \leq j \leq s_{i}$ are pairwise different injective vertices. Set $n_{k+1}=r+n_{1}$ and $s_{k+1}=s_{1}$. If $n_{i}-n_{i-1} \leq s_{i}$ for every $2 \leq i \leq k+1$, then $r=\sum_{i=2}^{k+1}\left(n_{i}-n_{i-1}\right) \leq \sum_{i=1}^{k} s_{i}$, which is a contradiction. Hence $n_{t}-n_{t-1}>s_{t}$ for some $t$ with $2 \leq t \leq k+1$. If $t \leq k$, then $\Gamma$ contains a path

$$
\begin{aligned}
\rho^{n_{k+1}} q_{1 t} & \rightarrow \rho^{n_{k+1}-n_{t}+n_{t-1}} q_{s_{t-1}, t-1} \rightarrow \cdots \rightarrow \rho^{n_{k+1}-n_{t}+n_{t-1}} q_{1, t-1} \rightarrow \cdots \\
& \rightarrow \rho^{n_{k+1}-n_{t}} q_{s_{1}, 1} \rightarrow \cdots \rightarrow \rho^{n_{k+1}-n_{t}} q_{11} \\
& \rightarrow \rho^{n_{k}-n_{t}} q_{s_{k}, k} \rightarrow \cdots \rightarrow \rho^{n_{k}-n_{t}} q_{1 k} \rightarrow \cdots \\
& \rightarrow \rho^{n_{t+1}-n_{t}} q_{1, t+1} \rightarrow q_{s_{t}, t} \rightarrow \cdots \rightarrow q_{1 t},
\end{aligned}
$$

where $n_{k+1}-\left(n_{k+1}-n_{t}+n_{t-1}\right)=n_{t}-n_{t-1}>s_{t}$. Therefore, with no loss of generality, we may assume that $n_{1}=0$ and $r-n_{k}>s_{1}$ in the path $(* *)$. In particular, $r-n_{k} \geq 2$. If $y$ is an immediate predecessor of $\rho^{r-n_{k}-2} q_{11}$, then $\rho y$ is an immediate predecessor of $\rho^{r-n_{k}-1} q_{11}$. Since $\Gamma$ is smooth, we have $\rho y=q_{s_{k}, k}$ or $\rho y=\rho^{r-n_{k}-1} z$, where $z$ is the immediate predecessor of $q_{11}$ in the path $(*)$. The first case does not happen since $q_{s_{k}, k}$ is injective. This shows that $\rho^{r-n_{k}-2} q_{11}$ has exactly one immediate predecessor $\rho^{r-n_{k}-2} z$ in $\Gamma$. Since $\Gamma$ is left stable, for each $1 \leq l \leq r-n_{k}-2$, the vertex $\rho^{l} q_{11}$ has exactly one immediate predecessor $\rho^{l} z$ in $\Gamma$. Since $s_{1}-1 \leq r-n_{k}-2$, we get $s_{1}$ co-rays $\rho^{l} P$ ending with $\rho^{l} q_{11}, l=0, \ldots, s_{1}-1$. Since $P$ is a co-ray, we 
see that $q_{11}$ has no successor in case $s_{1}>1$, and $q_{j 1}$ with $1<j<s_{1}$ has exactly one immediate successor $q_{j-1,1}$. Set $y_{1}=\rho^{r-1} q_{11}$ if $k=1$, and otherwise $y_{1}=\rho^{n_{2}-1} q_{12}$, that is $y_{1}$ is the non-injective immediate successor of $q_{s_{1}, 1}$. Let

$$
(* * *) \quad \cdots \rightarrow y_{n} \rightarrow y_{n-1} \rightarrow \cdots \rightarrow y_{2} \rightarrow y_{1}
$$

be the infinite sectional path with $y_{2} \neq q_{s_{1}, 1}$, and let

$$
\cdots \rightarrow z_{n} \rightarrow z_{n-1} \rightarrow \cdots \rightarrow z_{2} \rightarrow z_{1}=\rho^{s_{1}} q_{11}
$$

be the infinite sectional path $\rho^{s_{1}} P$. We shall construct a new valued translation quiver $(\mathcal{T}, \sigma)$ with trivial valuation. The vertices in $\mathcal{T}$ are the vertices in $\Gamma$ which do not belong to any of the corays $\rho^{l} P$, $l=0, \ldots, s_{1}-1$. The arrows in $\mathcal{T}$ are the arrows $a \rightarrow b$ in $\Gamma$ with $a, b \in \mathcal{T}$, plus the new arrows $z_{i} \rightarrow y_{i+1}, i=1,2, \cdots$. Finally, for each vertex $x$ in $\mathcal{T}$, we define $\sigma x=z_{i}$ in case $x=y_{i}$ for some $i \geq 1$; and otherwise, $\sigma x=\rho x$. It is easy to see that $\mathcal{T}$ is connected, left stable and smooth. Moreover, the path $(* * *)$ becomes a coray in $\mathcal{T}$ such that $\Gamma=\mathcal{T}\left[y_{1}, s_{1}\right]$. If $k=1$, then $z_{1}=\sigma y_{1}$ and

$$
y_{1}=\rho^{r-1} q_{11}=\rho^{r-s_{1}-1+s_{1}} q_{11}=\sigma^{r-s_{1}-1} z_{1}=\sigma^{r-s_{1}} y_{1} .
$$

Similarly, we have $y_{i}=\sigma^{r-s_{1}} y_{i}$ for $i \geq 2$. This implies that $\mathcal{T}$ is a stable tube of rank $r-s_{1}$, and hence $\Gamma$ is a coray tube. Assume that $k>1$. Then $\rho^{s_{1}} q_{11}=z_{1}=\sigma y_{1}=\sigma^{n_{2}} q_{21}$. Hence $\rho^{r-n_{2}} q_{11}=$ $\rho^{r-s_{1}-n_{2}+s_{1}} q_{11}=\sigma^{r-s_{1}} q_{12}$. In view of the path $(* *)$, we see that $\mathcal{T}$ contains a path

$$
\begin{aligned}
\sigma^{r-s_{1}} q_{12} & \rightarrow \sigma^{n_{k}-n_{2}} q_{s_{k}, k} \rightarrow \cdots \rightarrow \sigma^{n_{k}-n_{2}} q_{1 k} \rightarrow \cdots \\
& \rightarrow \sigma^{n_{3}-n_{2}} q_{13} \rightarrow q_{s_{2}, 2} \rightarrow \cdots \rightarrow q_{12}
\end{aligned}
$$

with $r-s_{1}>s_{2}+\cdots+s_{k}$ and $0<n_{3}-n_{2}<\cdots<n_{k}-n_{2}<r-s_{1}$. By the inductive hypothesis, $\mathcal{T}$ is a coray tube, and so is $\Gamma$. The proof of the theorem is completed.

\section{Auslander-Reiten COMPOnents}

Let $\mathcal{A}$ be a Krull-Schmidt $R$-category. We wish to have a general description of the shapes of the connected components of $\Gamma_{\mathcal{A}}$. This can be achieved by studying certain subquivers of $\Gamma_{\mathcal{A}}$. We say that a full subquiver $\Gamma$ of $\Gamma_{\mathcal{A}}$ is left (respectively, right) stable if it is closed under $\tau$ ( respectively, $\tau^{-}$), stable if it is left and right stable, and 
semi-stable if it is left or right stable. It is easy to see that semi-stable subquivers of $\Gamma_{\mathcal{A}}$ are locally finite. We start with stating a well known result; see, for example, [23, (2.2)].

5.1. Lemma. Let $\mathcal{A}$ be a connected Krull-Schmidt R-category. If $\Gamma$ is a finite connected component of $\Gamma_{\mathcal{A}}$ in which every object admits both a source morphism and a sink morphism, then $\Gamma=\Gamma_{\mathcal{A}}$.

If $\mathcal{A}$ is abelian, then every irreducible morphism is a monomorphism or an epimorphism, and consequently, $\Gamma_{\mathcal{A}}$ contains no loop. This is, however, not the case in general. On the other hand, if $\mathcal{A}$ is a connected left or right Auslander-Reiten category, then we shall be able to determine completely $\Gamma_{\mathcal{A}}$ if it contains a loop. For this purpose, we say that $\mathcal{A}$ is $A R$-exact if every Auslander-Reiten sequence is a short exact sequence. For instance, an abelian category is always ARexact; see (1.5). Moreover, we say that an indecomposable object $X$ in $\mathcal{A}$ is homogeneous if $\tau X=X$, and $\mathcal{A}$ is a homogeneous AuslanderReiten category if every indecomposable object in $\mathcal{A}$ is homogeneous.

5.2. Theorem. Let $\mathcal{A}$ be a connected left or right AuslanderReiten category. If $\Gamma_{\mathcal{A}}$ contains a loop, then $\mathcal{A}$ is an Auslander-Reiten category which is homogeneous but not $A R$-exact, and $\Gamma_{\mathcal{A}}$ is smooth of the following shape:

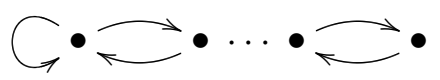

Proof. We consider only the case where $\mathcal{A}$ is a left AuslanderReiten category. Let $X \rightarrow X$ be a loop in $\Gamma_{\mathcal{A}}$. By Theorem 2.8, $X \rightarrow X \rightarrow X$ is not pre-sectional. Thus $\tau X=X$ and $d_{X X}^{\prime}=1$. Then $d_{X X}=d_{\tau X, X}=d_{X X}^{\prime}=1$. Let $f: X \rightarrow X$ be an irreducible morphism, which embeds in an Auslander-Reiten sequence:

$$
X \stackrel{\left(u, f_{1}\right)}{\longrightarrow} X \amalg Y_{1} \stackrel{\left(\begin{array}{l}
f \\
g
\end{array}\right)}{\longrightarrow} X .
$$

If $Y_{1}=0$, then the loop $X \rightarrow X$ alone is a connected component of $\Gamma_{\mathcal{A}}$. By Lemma $5.1, \Gamma_{\mathcal{A}}$ consists of this loop. Hence $\mathcal{A}$ is a homogeneous Auslander-Reiten category, which is not AR-exact since $f$ is not an epimorphism. 
Consider now the case where $Y_{1} \neq 0$. By Lemma 3.3, $d_{l}(f)<\infty$. Suppose that $Y_{1}$ has a summand $Z_{1} \amalg Z_{2}$ with $Z_{1}, Z_{2}$ indecomposable. By Lemmas 3.4(2) and 3.2, there exists an irreducible morphism $h$ : $X \rightarrow Z_{1} \amalg Z_{2}$ with $d_{l}(h)<d_{l}(f)$. By Lemma 3.7, there exists an irreducible morphism $h^{\prime}: \tau Z_{1} \amalg \tau Z_{2} \rightarrow X$ with $d_{l}\left(h^{\prime}\right)<d_{l}(h)$. In particular, $\tau Z_{1} \amalg \tau Z_{2}$ is a summand of $X \amalg Y_{1}$. Since $d_{X X}^{\prime}=1$, we have $X \neq Z_{i}$, and hence $X \neq \tau Z_{i}, i=1,2$. Therefore, $\tau Z_{1} \amalg \tau Z_{2}$ is a summand of $Y_{1}$. By Lemma 3.4(2), there exists an irreducible morphism $f^{\prime}: X \rightarrow X$ such that $d_{l}\left(f^{\prime}\right)<d_{l}\left(h^{\prime}\right)$. This yields $d_{l}\left(f^{\prime}\right)<$ $d_{l}(f)$, a contradiction to Lemma 3.6. Thus $Y_{1}$ is indecomposable and $Y_{1} \neq X$. Since $d_{l}(f)<\infty$, it follows from Lemma 3.5 that there exists a maximal integer $n \geq 1$ for which $\Gamma_{\mathcal{A}}$ contains a pre-sectional path

$$
(*) \quad Y_{n} \rightarrow Y_{n-1} \rightarrow \cdots \rightarrow Y_{1} \rightarrow Y_{0}=X .
$$

Moreover, the $Y_{i}$ with $0 \leq i \leq n$ are not pseudo-projective. For $0 \leq i \leq n$, denote by $\varepsilon_{i}$ the Auslander-Reiten sequence ending with $Y_{i}$. Setting $Y_{-1}=X$, we claim that $\varepsilon_{i}$ is of the form $Y_{i} \rightarrow Y_{i-1} \amalg Y_{i+1} \rightarrow$ $Y_{i}, i=0,1, \ldots, n-1$. Indeed, this is the case for $i=0$. Assume that there exists a minimal $0<r<n$ for which the claim does not hold. Then the middle term of $\varepsilon_{r-1}$ is $Y_{r-2} \amalg Y_{r}$. Since $\tau Y_{i}=Y_{i}$, for $-1 \leq i<r$, we have $Y_{r-2} \amalg Y_{r} \cong Y_{r-2} \amalg \tau Y_{r}$, and thus $\tau Y_{r} \cong Y_{r}$. Therefore, the middle term of $\varepsilon_{r}$ has a summand $Y_{r-1} \amalg Y_{r+1} \amalg Z_{r}$ with $Z_{r}$ indecomposable. By Lemmas 3.5 and 3.4(2), there exists an irreducible morphism $u_{r}: Y_{r} \rightarrow Y_{r+1} \amalg Z_{r}$ such that $d_{l}\left(u_{r}\right)<d_{l}(f)$, and by Lemma 3.7, there exists an irreducible morphism $v_{r}: \tau Y_{r+1} \amalg$ $\tau Z_{r} \rightarrow Y_{r}$ such that $d_{l}\left(v_{r}\right)<d_{l}\left(u_{r}\right)$. Since $\tau Y_{i} \cong Y_{i}, i=-1,0, \ldots, r$, there exists in $\Gamma_{\mathcal{A}}$ a pre-sectional path $X \rightarrow X \rightarrow Y_{1} \rightarrow \cdots \rightarrow Y_{r-1} \rightarrow$ $Y_{r}$. Since $Y_{r-1} \amalg \tau Y_{r+1} \amalg \tau Z_{r}$ is a summand of the middle term of $\varepsilon_{r}$, by Lemma 3.5, there exists an irreducible morphism $h: X \rightarrow X$ such that $d_{l}(h)<d_{l}\left(v_{r}\right)$, and hence $d_{l}(h)<d_{l}(f)$, which is again contrary to Lemma 3.6. Our claim is established. In particular, $Y_{n-2} \amalg \tau Y_{n}$ is isomorphic to $Y_{n-2} \amalg Y_{n}$, the middle term of $\varepsilon_{n-1}$. Hence $\tau Y_{n} \cong Y_{n}$. It follows from the maximality of $n$ that $\varepsilon_{n}$ is of the form $Y_{n} \rightarrow Y_{n-1} \rightarrow$ $Y_{n}$. Therefore, the full translation subquiver of $\Gamma_{\mathcal{A}}$ generated by the $Y_{i}$ with $i \leq 0 \leq n$ is a connected component. By Lemma 5.1, the $Y_{i}$ with $i \leq 0 \leq n$ are the indecomposable objects in $\mathcal{A}$ up to isomorphism. In particular, $\mathcal{A}$ is a homogeneous Auslander-Reiten category.

Next, we claim that the $Y_{i}$ with $0 \leq i \leq n$ are pairwise nonisomorphic. If this is not the case, then there exists some minimal $s$ with $0 \leq s<n$ such that $Y_{s} \cong Y_{t}$ for some $t$ with $s<t \leq n$. Since 
the middle term of $\varepsilon_{n}$ is indecomposable, we have $t<n$. If $s=0$, then $t>1$ and $Y_{t+1}=X$ or $Y_{t+1}=Y_{1}$. By Theorem 2.8, $Y_{t+1}=X$, and thus $\Gamma_{\mathcal{A}}$ has a pre-sectional path

$$
X \rightarrow X \rightarrow Y_{t-1} \rightarrow \cdots \rightarrow Y_{1} \rightarrow X \rightarrow X
$$

which is contrary to Theorem 2.8. Thus $0<s<t<n$, and then $Y_{s-1} \amalg Y_{s+1} \cong Y_{t-1} \amalg Y_{t+1}$. Therefore, $Y_{s-1} \cong Y_{t-1}$ or $Y_{s-1} \cong Y_{t+1}$, which contradicts the minimality of $s$. Our second claim is proved. In particular, $\Gamma_{\mathcal{A}}$ is trivially valued. This shows that $\Gamma_{\mathcal{A}}$ is of the shape as stated in the theorem. It remains to show that $\mathcal{A}$ is not AR-exact. Setting $f_{0}=f$ and applying repeatedly Theorem 1.4(2), we find Auslander-Reiten sequences

$$
Y_{i} \stackrel{\left(p_{i}, f_{i+1}\right)}{\longrightarrow} Y_{i-1} \amalg Y_{i+1} \stackrel{\left(\begin{array}{l}
f_{i} \\
g_{i}
\end{array}\right)}{\longrightarrow} Y_{i}, i=0,1, \ldots, n-1,
$$

and $Y_{n} \stackrel{p_{n}}{\longrightarrow} Y_{n-1} \stackrel{f_{n}}{\longrightarrow} Y_{n}$. Dually, setting $u_{0}=f$, we get AuslanderReiten sequences

$$
Y_{i} \stackrel{\left(q_{i}, u_{i}\right)}{\longrightarrow} Y_{i+1} \amalg Y_{i-1} \stackrel{\left(\begin{array}{c}
u_{i+1} \\
h_{i}
\end{array}\right)}{\longrightarrow} Y_{i}, i=0,1, \ldots, n-1,
$$

and $Y_{n} \stackrel{u_{n}}{\longrightarrow} Y_{n-1} \stackrel{h_{n}}{\longrightarrow} Y_{n}$. This yields

$$
f_{n}\left(g_{n-1} \cdots g_{1} g_{0} h_{0} h_{1} \cdots h_{n}\right)=p_{n-1} \cdots p_{1} p_{0} q_{0} q_{1} \cdots q_{n-1} u_{n} h_{n}=0 .
$$

Since $g_{n-1} \cdots g_{1} g_{0} h_{0} h_{1} \cdots h_{n} \neq 0$ by Lemma $2.7(1), f_{n}$ is not an epimorphism. That is, $\varepsilon_{n}$ is not a short exact sequence. The proof of the theorem is completed.

REMARK. The above result was established by Xiao and Zhu for triangulated categories having Auslander-Reiten triangles; see [27].

ExAmple. (1) Let $\mathcal{A}$ be a Krull-Schmidt category over a field $k$ with an unique indecomposable object $X$ whose endomorphism algebra is the quotient of $k[x]$ modulo $x^{2}$. Setting $f=\bar{x} \in \operatorname{End}(X)$, we see that $X \stackrel{f}{\longrightarrow} X \stackrel{f}{\longrightarrow} X$ is the unique Auslander-Reiten sequence in $\mathcal{A}$. Therefore, $\mathcal{A}$ is a homogeneous Auslander-Reiten category with $\Gamma_{\mathcal{A}}$ consisting of a single loop at $X$.

(2) Let $S$ be a complete discrete valuation commutative ring with quotient field $K$. In [26], Wiedemann gave a complete description of the two-sided indecomposable $S$-orders $\Lambda$ in a semi-simple $K$-algebra which admit an irreducible map from some indecomposable $\Lambda$-lattice 
to itself. Moreover, the Auslander-Reiten quiver of such an order $\Lambda$ is of the shape as stated in Theorem 5.2.

5.3. Lemma. Let $\mathcal{A}$ be a left Auslander-Reiten category, and let $\Gamma$ be a connected left stable subquiver of $\Gamma_{\mathcal{A}}$ containing an infinite sectional path

$$
\cdots \rightarrow X_{n} \rightarrow \cdots \rightarrow X_{1} \rightarrow X_{0} .
$$

If the path contains infinitely many arrows of finite global left degree, then $\Gamma$ is smooth and contains no double infinite sectional path.

Proof. Assume that the path contains infinitely many arrows of finite global left degree. For $i, j \geq 0$, by Lemma 3.11, the infinite sectional path in $\Gamma$ ending with $\tau^{j} X_{i}$ generated by the objects $\tau^{j} X_{n}$ with $n \geq i$ contains infinitely many arrows of finite global left degree. By Lemma 3.10, $\tau^{j} X_{i}$ has at most two immediate predecessors in $\Gamma$.

Let $X$ be an object in $\Gamma$. We claim that there exists some $r \geq 0$ such that $\tau^{r} X$ is the ending-point of an infinite sectional path in $\Gamma$ with infinitely many arrows of finite global left degree. Indeed, by Lemma 4.1, there exist $r, s \geq 0$ such that either $\tau^{r} X=\tau^{s} X_{0}$ or $\Gamma$ contains a sectional path

$$
\tau^{r} X \rightarrow Y_{t-1} \rightarrow \cdots \rightarrow Y_{1} \rightarrow Y_{0}=\tau^{s} X_{0}
$$

with $t \geq 1$. If $Y_{1}=\tau^{s} X_{1}$, then $\tau^{r} X=\tau^{s} X_{t}$, and the claim follows. Otherwise, $\Gamma$ contains an infinite sectional path

$$
\cdots \rightarrow \tau^{s+t} X_{1} \rightarrow \tau^{s+t} X_{0} \rightarrow \tau^{t-1} Y_{1} \rightarrow \cdots \rightarrow \tau Y_{t-1} \rightarrow \tau^{r} X
$$

which, by Lemma 3.11, contains infinitely many arrows of finite global left degree. The claim is established. Since $\Gamma$ is left stable, it follows from Lemma 3.10 that $X$ has at most two immediate predecessors and at most two immediate successors in $\Gamma$. Let now $X \rightarrow Y$ be an arrow in $\Gamma$. In view of our claim, it is easy to see that there exists some $r \geq 0$ such that $\Gamma$ contains an infinite sectional path which ends with the arrow $\tau^{r} X \rightarrow \tau^{r} Y$ or $\tau^{r+1} Y \rightarrow \tau^{r} X$, and has infinitely many arrows of finite global left degree. It then follows from Lemma 3.10 that $X \rightarrow Y$ is trivially valued. This shows that $\Gamma$ is smooth. Finally, suppose that $\Gamma$ contains a double infinite sectional path $P$ as follows:

$$
\cdots \rightarrow Z_{i} \rightarrow \cdots \rightarrow Z_{1} \rightarrow Z_{0} \rightarrow \cdots \rightarrow Z_{-i} \rightarrow \cdots
$$

Then there exists some $r \geq 0$ such that $\tau^{r} Z_{1} \rightarrow \tau^{r} Z_{0}$ or $\tau^{r+1} Z_{0} \rightarrow$ $\tau^{r} Z_{1}$ is the ending arrow of an infinite sectional path $Q$ in $\Gamma$ which has infinitely many arrows of finite global left degree. If $Q$ ends with 
$\tau^{r+1} Z_{0} \rightarrow \tau^{r} Z_{1}$, by applying Lemma 3.8 to $Q$ and the path generated by the $\tau^{r} Z_{n}$ with $n \geq 1$, we see that $Q$ has no arrows of finite global left degree, a contradiction. Thus $Q$ ends with $\tau^{r} Z_{1} \rightarrow \tau^{r} Z_{0}$. Since $\Gamma$ is a smooth, $Q$ coincides with the path generated by the $\tau^{r} Z_{n}$ with $n \geq 0$, which is contained in the double infinite sectional path $\tau^{r} P$, a contradiction to Corollary 3.10. The proof of the lemma is completed.

5.4. Lemma. Let $\mathcal{A}$ be a left Auslander-Reiten category, and let $\Gamma$ be a connected left stable subquiver of $\Gamma_{\mathcal{A}}$. If $\Gamma$ contains oriented cycles but no $\tau$-periodic object, then $\Gamma$ contains an infinite sectional path

$$
X_{1} \rightarrow \cdots \rightarrow X_{s} \rightarrow \tau^{r} X_{1} \rightarrow \cdots \rightarrow \tau^{r} X_{s} \rightarrow \tau^{2 r} X_{1} \rightarrow \cdots,
$$

where $r, s>0$ and the $\tau$-orbits of $X_{1}, \ldots, X_{s}$ are pairwise different.

Proof. Suppose that $\Gamma$ contains oriented cycles but no $\tau$-periodic object. By Theorem 5.2, $\Gamma$ contains no loop. Let $X$ be an object lying on an oriented cycle. Since $X$ is not $\tau$-periodic, by Lemma 4.1(2), $\Gamma$ contains a sectional path

$$
\text { (*) } \quad X=X_{1} \rightarrow \cdots \rightarrow X_{s} \rightarrow X_{s+1},
$$

where $s \geq 1$ and $X_{s+1}=\tau^{r} X_{1}$ for some $r \geq 0$. We assume that $s$ is the minimal length of such sectional paths in $\Gamma$. Suppose that $r=0$. Then $s>1$. By Theorem 2.8, $X_{s}=\tau X_{2}$. Since $X_{2}$ is not $\tau$-periodic, we have $s>2$, which contradicts the minimality of $s$. This shows that $r>0$. Assume now that there exists some $1 \leq i<j \leq s$ such that $X_{i}$ and $X_{j}$ lie in the same $\tau$-orbit. By the minimality of $s$, we get $X_{j}=\tau^{-p} X_{i}$ with $p>0$. Since $X_{s} \neq \tau^{r+1} X_{2}$, we have a sectional path $X_{j} \rightarrow \cdots \rightarrow X_{s} \rightarrow \tau^{r} X_{1} \rightarrow \cdots \rightarrow \tau^{r} X_{i}$ of length $s-(j-i)$ with $\tau^{r} X_{i}=\tau^{r+p} X_{j}$, which is contrary again to the minimality of $s$. This proves that the $X_{i}$ with $1 \leq i \leq s$ lie in pairwise different $\tau$-orbits. Applying repeatedly $\tau^{r}$ to the path $(*)$, we get an infinite path

$$
X_{1} \rightarrow \cdots \rightarrow X_{s} \rightarrow \tau^{r} X_{1} \rightarrow \cdots \rightarrow \tau^{r} X_{s} \rightarrow \tau^{2 r} X_{1} \rightarrow \cdots,
$$

which is sectional since the $X_{i}$ are not $\tau$-periodic. The proof of the lemma is completed.

We are now ready to state our main result on a general description of the shapes of semi-stable subquivers of $\Gamma_{\mathcal{A}}$. This generalizes the results on semi-stable components of the Auslander-Reiten quiver of an artin algebra which can be found in $[11,19,28]$. 
5.5. TheOREM. Let $\mathcal{A}$ be a Krull-Schmidt R-category, and let $\Gamma$ be a connected left stable subquiver of $\Gamma_{\mathcal{A}}$.

(1) If $\Gamma$ contains no oriented cycle, then $\Gamma$ embeds in $\mathbb{Z} \Delta$, where $\Delta$ is a valued quiver with an unique sink but no oriented cycle.

(2) If $\Gamma$ contains $\tau$-periodic objects, then $\Gamma$ is a stable tube or $\Gamma \cong$ $\mathbb{Z} \Delta / G$, where $\Delta$ is a valued Dynkin quiver and $G$ is an automorphism group of $\mathbb{Z} \Delta$ containing a positive power of the translation.

(3) If $\Gamma$ contains oriented cycles but no $\tau$-periodic object, then $\Gamma$ is smooth and not stable, and contains an infinite sectional path

$$
\cdots \rightarrow \tau^{2 r} X_{1} \rightarrow \tau^{r} X_{s} \rightarrow \cdots \rightarrow \tau^{r} X_{1} \rightarrow X_{s} \rightarrow \cdots \rightarrow X_{1},
$$

where $r>s>0$, and $X_{1}, \ldots, X_{s}$ form a complete set of representatives of the $\tau$-orbits in $\Gamma$.

Proof. Let $\mathcal{B}$ be the full additive subcategory of $\mathcal{A}$ generated by the objects which have no summands isomorphic to objects lying in $\Gamma$. By Lemma 1.9(2), the ideal $\mathcal{I}$ of the morphisms factoring through objects in $\mathcal{B}$ is admissible. We then deduce from Proposition 1.8 that $\Gamma$ is the Auslander-Reiten quiver of $\mathcal{A} / I$. In particular, $\mathcal{A} / I$ is a left Auslander-Reiten category. Hence we may assume with no loss of generality that $\mathcal{A}$ is a connected left Auslander-Reiten category.

Statement (1) follows immediately from Theorem 4.2. For proving Statement (2), assume that $\Gamma$ contains a $\tau$-periodic object. Since $\Gamma$ is locally finite, the objects in $\Gamma$ are all $\tau$-periodic. If $\Gamma$ contains a loop then, by Theorem 5.2, $\Gamma$ is homogeneous, smooth, and of the shape

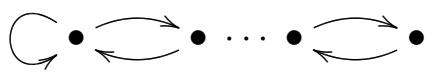

Let $n$ be the number of vertices in $\Gamma$, and consider the trivially valued quiver

$$
A_{2 n}: \quad 2 n \longrightarrow \cdots \longrightarrow n+1 \longrightarrow n \longrightarrow \cdots \longrightarrow 1 .
$$

Denote by $\sigma$ the unique automorphism of $\mathbb{Z} A_{2 n}$ satisfying the condition that $\sigma(i, j)=(2 n-i+1, j+2 n-i)$, for $1 \leq i \leq 2 n$ and $j \in \mathbb{Z}$, and let $G$ be the group generated $\sigma$ and the translation of $\mathbb{Z} A_{2 n}$. It is then easy to see that $\Gamma \cong \mathbb{Z} A_{2 n} / G$. Consider next the case where $\Gamma$ contains no loop. Choose an object $M$ in $\Gamma$. Let $r$ be the $\tau$-period of $M$ and set $N=\amalg_{i=1}^{r} \tau^{i} M$. For each object $X$ in $\Gamma$, put $d(X)=\ell_{R}\left(\operatorname{Hom}_{\mathcal{A}}(X, N)\right)$. We claim that $d(X)>0$ for every object $X$ in $\Gamma$. Being $\tau$-periodic, $\Gamma$ contains a non-trivial 
path from $X$ to $M$. If $X=\tau^{i} M$ for some $i>0$, then it is evident that $\operatorname{Hom}_{\mathcal{A}}(X, N) \neq 0$. Otherwise, by Lemma 4.1(2), $\Gamma$ has a sectional path $X=M_{0} \rightarrow \cdots \rightarrow M_{s} \rightarrow \tau^{t} M$ with $s, t \geq 0$. Hence $\operatorname{Hom}_{\mathcal{A}}\left(X, \tau^{t} M\right) \neq 0$ by Lemma 2.7(1). This establishes our claim. If $X \stackrel{f}{\longrightarrow} Y \stackrel{g}{\longrightarrow} Z$ is an Auslander-Reiten sequence in $\mathcal{A}$, then

$$
\operatorname{Hom}_{\mathcal{A}}(Z, N) \stackrel{g_{*}}{\longrightarrow} \operatorname{Hom}_{\mathcal{A}}(Y, N) \stackrel{f_{*}}{\longrightarrow} \operatorname{rad}(X, N) \longrightarrow 0
$$

is an exact sequence in the category of finitely generated $R$-modules. Therefore,

$$
d(Y)=\ell_{R}\left(\operatorname{Hom}_{\mathcal{A}}(Y, N)\right) \leq \ell_{R}(\operatorname{rad}(X, N))+d(Z) \leq d(X)+d(Z),
$$

where the last inequality is strict whenever $X$ is a summand of $N$. This shows that $d$ is a subadditive function on $\Gamma$ which is not additive in the sense of [11]. In this case, Statement (2) follows from the theorem stated in [11, Section 2]; see also [5, (VII.3.3)]. For proving Statement (3), suppose that $\Gamma$ contains oriented cycles but no $\tau$ periodic object. By Lemma 5.4, $\Gamma$ contains an infinite sectional path

$$
Y_{1} \rightarrow \cdots \rightarrow Y_{s} \rightarrow \tau^{t} Y_{1} \rightarrow \cdots \rightarrow \tau^{t} Y_{s} \rightarrow \tau^{2 r} Y_{1} \rightarrow \cdots,
$$

where $t, s \geq 1$, and $Y_{1}, \ldots, Y_{s}$ lie in pairwise different $\tau$-orbits. Setting $X_{i}=\tau^{i-1} Y_{i}, i=1, \ldots, s$, we get an infinite sectional path

$$
\cdots \rightarrow \tau^{2 r} X_{1} \rightarrow \tau^{r} X_{s} \rightarrow \cdots \rightarrow \tau^{r} X_{1} \rightarrow X_{s} \rightarrow \cdots \rightarrow X_{1}
$$

in $\Gamma$, where $r=t+s>s$. For each $j \geq 0$, the path

$$
P_{j}: \quad \tau^{(j+1) r} X_{1} \rightarrow \tau^{j r} X_{s} \rightarrow \cdots \rightarrow \tau^{j r} X_{1}
$$

induces an oriented cycle from $\tau^{(j+1) r} X_{1}$ to $\tau^{(j+1) r} X_{1}$ passing through $\tau^{j r+s} X_{1}$ which, by Lemma 3.3 , contains an arrow of finite left degree. Thus $P_{j}$ contains an arrow of finite global left degree. By Lemma 5.3, $\Gamma$ is smooth and contains no double infinite sectional path. Therefore, $X_{1}, \ldots, X_{s}$ are not all stable. Moreover, by Lemma 4.3, every object in $\Gamma$ lies in one of the $\tau$-orbits of $Y_{1}, \ldots, Y_{s}$. That is, $X_{1}, \ldots, X_{s}$ form a complete set of representatives of $\tau$-orbits in $\Gamma$. The proof of the theorem is completed.

As an application, we shall describe the connected stable subquivers of $\Gamma_{\mathcal{A}}$. Note that the $\tau$-periodic ones have already been described in Theorem 5.5(2). 
5.6. Corollary. If $\mathcal{A}$ is a Krull-Schmidt R-category, then the connected stable subquivers of $\Gamma_{\mathcal{A}}$ are either $\tau$-periodic or of shape $\mathbb{Z} \Delta$, where $\Delta$ is a locally finite valued quiver with no oriented cycle.

Proof. Let $\Gamma$ be a connected stable subquiver of $\Gamma_{\mathcal{A}}$. If $\Gamma$ is not $\tau$-periodic then, by Theorem 5.5(3), $\Gamma$ contains no oriented cycle. By Theorem 4.2, $\Gamma$ contains a section $\Delta$. Since $\Gamma$ is locally finite and stable, $\Delta$ is locally finite and the canonical embedding $\Gamma \rightarrow \mathbb{Z} \Delta$, which sends $\tau^{n} X$ to $(n, X)$, is an isomorphism; see [20, (2.3)]. The proof of the corollary is completed.

Let $\Gamma$ be a connected component of $\Gamma_{\mathcal{A}}$. By abuse of language, we shall say that an Auslander-Reiten sequence $X \rightarrow Y \rightarrow Z$ lies in $\Gamma$ if $X$, or equivalently $Z$, lies in $\Gamma$. Moreover, we say that $\Gamma$ is left regular or right regular if $\Gamma$, as a subquiver of $\Gamma_{\mathcal{A}}$, is left stable or right stable, respectively. Furthermore, $\Gamma$ is called semi-regular if it is left or right regular, and regular if it is left and right regular. The semi-regular components of $\Gamma_{\mathcal{A}}$ which are $\tau$-periodic or contain no oriented cycle are well described in Theorem 5.5. However, the description for those containing oriented cycles but no $\tau$-periodic object is less satisfactory. We can do a better job if the Auslander-Reiten sequences lying in the component are all short exact sequences; compare [19, (2.5)].

5.7. TheOREm. Let $\mathcal{A}$ be a Krull-Schmidt R-category, and let $\Gamma$ be a semi-regular component of $\Gamma_{\mathcal{A}}$ which contains oriented cycles but no $\tau$-periodic object. If the Auslander-Reiten sequences lying in $\Gamma$ are all short exact sequences, then $\Gamma$ is a ray tube or a co-ray tube.

Proof. We consider only the case where $\Gamma$ is a left regular component in which every Auslander-Reiten sequence is short exact. Let $\mathcal{B}$ be the full additive subcategory of $\mathcal{A}$ generated by the objects whose indecomposables summands are isomorphic to objects lying in $\Gamma$. It is clear that $\mathcal{B}$ is a Krull-Schmidt $R$-category. Let $X$ be an indecomposable object in $\mathcal{B}$ lying in $\Gamma$. Since $\Gamma$ is left regular, there exists an Auslander-Reiten short exact sequence $Z \rightarrow Y \rightarrow X$ in $\mathcal{A}$. Since $X, Y, Z$ all lie in $\mathcal{B}$, it is easy to verify that $Z \rightarrow Y \rightarrow X$ is an Auslander-Reiten short exact sequence in $\mathcal{B}$. This implies that $\mathcal{B}$ is a left Auslander-Reiten category which is AR-exact and has $\Gamma$ as its Auslander-Reiten quiver. As a consequence, we may assume with no loss of generality that $\mathcal{A}$ is a left Auslander-Reiten category. By 
Theorem 5.5(3), $\Gamma$ is smooth and contains an infinite sectional path

$$
\cdots \rightarrow \tau^{2 r} X_{1} \rightarrow \tau^{r} X_{s} \rightarrow \cdots \rightarrow \tau^{r} X_{1} \rightarrow X_{s} \rightarrow \cdots \rightarrow X_{1}
$$

with infinitely many arrows of finite global left degree, where $r>s$, and $X_{1}, \ldots, X_{s}$ are not all stable and form a complete set of representatives of the $\tau$-orbits in $\Gamma$. For convenience, rewrite $(*)$ as follows:

$$
\cdots \rightarrow X_{2 s+1} \rightarrow X_{2 s} \rightarrow \cdots \rightarrow X_{s+1} \rightarrow X_{s} \rightarrow \cdots \rightarrow X_{1},
$$

where $X_{j s+i}=\tau^{r j} X_{i}$ with $j \geq 0$ and $1 \leq i \leq s$. For each $q \geq 0$, we claim that if $\tau^{-q} X_{i}$ is defined for some $i \geq 1$, then $\tau^{-q} X_{i+1}$ is also defined. If this is not the case, then we can find a minimal $m>0$ for which there exists some $n \geq 1$ such that $\tau^{-m} X_{n}$ is defined but $\tau^{-m} X_{n+1}$ is not. By the minimality of $m$, there exists in $\Gamma$ an infinite sectional path

$$
(* *) \quad \cdots \rightarrow \tau^{-m+1} X_{i} \rightarrow \cdots \rightarrow \tau^{-m+1} X_{n+1} \rightarrow \tau^{-m+1} X_{n} .
$$

Set $Y_{0}=\tau^{-m} X_{n}$, and let $\tau Y_{0} \stackrel{f_{1}}{\longrightarrow} Y_{1} \stackrel{g_{1}}{\longrightarrow} Y_{0}$ be an AuslanderReiten sequence in $\mathcal{A}$, which is a short exact sequence by assumption. In particular, $f_{1}$ is a monomorphism. Since $\Gamma$ is smooth and left regular while $\tau^{-m} X_{n+1}$ is not defined, $Y_{1}$ is indecomposable and $\tau Y_{1} \not \approx \tau^{-m+1} X_{n+1}$. Since $(*)$ contains infinitely many arrows of finite global left degree, so does (**). By Lemma 3.8, $\Gamma$ contains no infinite sectional path ending with $Y_{1} \rightarrow Y_{0}$. Hence there exists a maximal integer $t>0$ such that $\Gamma$ contains a sectional path

$$
Y_{t} \rightarrow \cdots \rightarrow Y_{1} \rightarrow Y_{0}
$$

Since $\Gamma$ is smooth, the middle term of the Auslander-Reiten sequence ending with $Y_{j}$ is $\tau Y_{j-1} \amalg Y_{j+1}, j=1, \ldots, t-1$. By the maximality of $t$, the middle term of the Auslander-Reiten sequence ending with $Y_{t}$ is $\tau Y_{t-1}$. Choose irreducible morphisms $g_{j}: Y_{j} \rightarrow Y_{j-1}, h_{j}: \tau Y_{j} \rightarrow$ $\tau Y_{j-1}$, and $f_{j}: \tau Y_{j-1} \rightarrow Y_{j}, j=1, \ldots, t$, such that $\tau Y_{t} \stackrel{h_{t}}{\longrightarrow} \tau Y_{t-1} \stackrel{f_{t}}{\longrightarrow}$ $Y_{t}$ and

$$
\tau Y_{j} \stackrel{\left(f_{j+1}, h_{j}\right)}{\longrightarrow} Y_{j+1} \amalg \tau Y_{j-1} \stackrel{\left(\begin{array}{c}
g_{j+1} \\
f_{j}
\end{array}\right)}{\longrightarrow} Y_{j}, j=1, \ldots, t-1,
$$

are Auslander-Reiten sequences in $\mathcal{A}$. Then $h_{n} \cdots h_{1} f_{1}=0$. Since $f_{1}$ is a monomorphism, $h_{n} \cdots h_{1}=0$. This is contrary to Lemma 2.7(1). Our claim is established. If $X_{i}$ is stable for some $1 \leq i \leq s$, then it follows from the claim that $X_{j}$ is stable for all $j \geq i$. As a consequence, the $X_{i}$ with $1 \leq i \leq s$ are all stable, a contradiction. Therefore, 
$X_{i}=\tau^{m_{i}} I_{i}, i=1, \ldots, s$, where $I_{i}$ is some object in $\Gamma$ for which $\tau^{-}$ is not defined. Using the claim again, we have $m_{s} \geq \cdots \geq m_{2} \geq m_{1}$. By Theorem 4.4, $\Gamma$ is a coray tube. The proof of the theorem is completed.

\section{Specialization to triangulated CATEgories}

In this section, we shall apply the previously developed theory to triangulated categories. Recall first that an additive category is triangulated if it is equipped with an automorphism $T$, called shift functor, and a class of sextuples $X \stackrel{f}{\longrightarrow} Y \stackrel{g}{\longrightarrow} Z \stackrel{h}{\longrightarrow} T X$, called exact triangles, satisfying the axioms TR1, TR2, TR3 and TR4 stated in [10, (1.1)]. A classical example of a triangulated category is given by the derived category $D^{b}(A)$ of the bounded complexes of finitely generated modules over an artin algebra $A$; see [10]. We call a triangulated category triangle-connected if it can not be decomposed as a product of two non-zero triangulated categories; and triangle-simple if it admits exactly one indecomposable object up to isomorphism and shift, and the non-zero morphisms between indecomposable objects are isomorphisms.

For the rest of the paper, $\mathcal{A}$ denotes a Krull-Schmidt triangulated $R$-category. The shift of an object $X$ by an integer $n$ is written as $X[n]$. Consider an exact triangle $X \stackrel{f}{\longrightarrow} Y \stackrel{g}{\longrightarrow} Z \stackrel{h}{\longrightarrow} X[1]$ in $\mathcal{A}$. It is important to note that $X \stackrel{f}{\longrightarrow} Y \stackrel{g}{\longrightarrow} Z$ is a pseudo-exact sequence; see $[10,(1.2)]$. Moreover, $f$ is a source morphism if and only if $g$ is a sink morphism; see [10, (4.5)], and in this case, the exact triangle is called an Auslander-Reiten triangle; compare [10, (4.1)]. The connection between the Auslander-Reiten sequences and the Auslander-Reiten triangles in $\mathcal{A}$ is established in the following easy lemma.

6.1. Lemma. A sequence $X \stackrel{f}{\longrightarrow} Y \stackrel{g}{\longrightarrow} Z$ with $Y \neq 0$ in $\mathcal{A}$ is an Auslander-Reiten sequence if and only if it can be embedded in an Auslander-Reiten triangle $X \stackrel{f}{\longrightarrow} Y \stackrel{g}{\longrightarrow} Z \stackrel{h}{\longrightarrow} X[1]$.

Proof. The sufficiency is evident. Let $X \stackrel{f}{\longrightarrow} Y \stackrel{g}{\longrightarrow} Z$ be an Auslander-Reiten sequence in $\mathcal{A}$. By the axiom TR1 stated in $[10$, 
(1.1)], $f$ embeds in an exact triangle $X \stackrel{f}{\longrightarrow} Y \stackrel{g^{\prime}}{\longrightarrow} Z^{\prime} \stackrel{h}{\longrightarrow} X[1]$, which is an Auslander-Reiten triangle since $f$ is a source morphism; see $[10,(4.5)]$. In particular, $X \stackrel{f}{\longrightarrow} Y \stackrel{g^{\prime}}{\longrightarrow} Z^{\prime}$ is an Auslander-Reiten sequence. By the uniqueness stated in Theorem 1.4(1), we get a commutative diagram

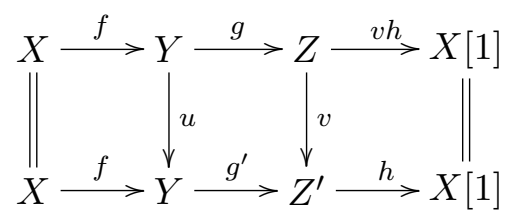

in $\mathcal{A}$, where $u, v$ are isomorphisms. Therefore, the upper row is an Auslander-Reinten triangle. The proof of the lemma is completed.

Note that a triangulated category (for instance, the bounded derived category of a simple algebra) may have Auslander-Reiten triangles of the form $X \rightarrow 0 \rightarrow Z \rightarrow X[1]$. However, this occurs rarely according to the next result.

6.2. Proposition. If $\mathcal{A}$ is triangle-connected, then the following statements are equivalent.

(1) $\mathcal{A}$ is triangle-simple.

(2) $\mathcal{A}$ has a pseudo-projective object.

(3) $\mathcal{A}$ has a pseudo-injective object. $X[1]$.

(4) $\mathcal{A}$ has an Auslander-Reiten triangle of the form $X \rightarrow 0 \rightarrow Z \rightarrow$

Proof. Let $\mathcal{A}$ be triangle-connected. Assume first that $\mathcal{A}$ is trianglesimple. Choose an indecomposable object $X$ in $\mathcal{A}$. By the definition of triangle-simplicity, all non-zero morphisms $Y \rightarrow X$ are retractions and all non-zero morphisms $X \rightarrow Y$ are sections. Thus $0 \rightarrow X$ is a sink monomorphism while $X \rightarrow 0$ is a source epimorphism. That is, $X$ is pseudo-projective as well as pseudo-injective.

Assume next that $\mathcal{A}$ has a sink monomorphism $g: Y \rightarrow Z$. Then $g$ embeds in an Auslander-Reiten triangle $X \stackrel{f}{\longrightarrow} Y \stackrel{g}{\longrightarrow} Z \stackrel{h}{\longrightarrow} X[1]$; see $[10,(1.3),(4.5)]$. Moreover, $f=0$ since $g$ is a monomorphism. By Lemma 1.1, we get $Y=0$. This proves that (2) implies (4). Dually, one can show that (3) implies (4). 
Finally, let $X \rightarrow 0 \rightarrow Z \stackrel{h}{\rightarrow} X[1]$ be an Auslander-Reiten triangle in $\mathcal{A}$. Then $X \rightarrow 0$ is a source morphism. Since $0 \rightarrow Z \stackrel{h}{\longrightarrow} X[1] \rightarrow 0$ is an exact triangle in $\mathcal{A}$; see $[10,(1.1)], h$ is an isomorphism. Thus $0 \rightarrow X[1]$ is a sink morphism. As a consequence, $X[n] \rightarrow 0$ is a source morphism and $0 \rightarrow X[n]$ is a sink morphism, for every integer $n$. Therefore, the non-zero morphisms $X[n] \rightarrow M$ and $N \rightarrow X[n]$ with $M, N$ indecomposable are isomorphisms. It is easy to see that the full additive subcategory $\mathcal{B}$ of $\mathcal{A}$ generated by the $X[n]$ is a trianglecomponent of $\mathcal{A}$. Since $\mathcal{A}$ is triangle-connected, we get $\mathcal{B}=\mathcal{A}$. In particular, the $X[n]$ are the indecomposable objects in $\mathcal{A}$ up to isomorphism. This implies $\mathcal{A}$ is triangle-simple. The proof of the proposition is completed.

From now on, we assume that $\mathcal{A}$ is triangle-connected but not triangle-simple. If $\mathcal{A}$ is a left or right Auslander-Reiten category, it follows from Lemma 6.1 and Proposition 6.2 that the AuslanderReiten quiver of $\mathcal{A}$ defined in this paper coincides with the one defined in [10]. Note that if $R$ is an algebraically closed field, then $\mathcal{A}$ is a left Auslander-Reiten category if and only if $\mathcal{A}$ admits a right Serre functor, that is an additive endofunctor $F: \mathcal{A} \rightarrow \mathcal{A}$ such that $\operatorname{Hom}_{\mathcal{A}}(X, Y) \cong \operatorname{Hom}_{\mathcal{A}}(Y, F X)^{*}$ which are natural for all $X, Y$ in $\mathcal{A}$, where $U^{*}=\operatorname{Hom}_{R}(U, R)$. Dually, $\mathcal{A}$ is a right Auslander-Reiten category if and only if $\mathcal{A}$ admits a left Serre functor; see [22].

6.3. Theorem. Let $\mathcal{A}$ be a Krull-Schmidt triangulated $R$-category which is triangle-connected but not triangle-simple. If $\mathcal{A}$ is a left (respectively, right) Auslander-Reiten category, then every component of $\Gamma_{\mathcal{A}}$ is left (respectively, right) regular, which is either $\tau$-periodic or a coray (respectively, ray) tube, or embeds in $\mathbb{Z} \Delta$, where $\Delta$ is a locally finite valued quiver with no oriented cycle.

Proof. Assume that $\mathcal{A}$ is a left Auslander-Reiten category, and let $\Gamma$ be a component of $\Gamma_{\mathcal{A}}$ which is left regular By Proposition 6.2. It suffices to consider the case where $\Gamma$ contains oriented cycles but no $\tau$-periodic object. By Theorem 5.5(3), $\Gamma$ is smooth and not stable. We want to show that $\Gamma$ is a coray tube. In view of the proof of Theorem 5.7, we need only to show the following statement: given an infinite sectional path

$$
\cdots \rightarrow X_{n} \rightarrow \cdots \rightarrow X_{2} \rightarrow X_{1}
$$


in $\Gamma$ with infinitely many arrows of finite global left degree, if $\tau^{-p} X_{i}$ is defined for some $i, p>0$, then $\tau^{-p} X_{i+1}$ is also defined. Assume that this is false. Let $m>0$ be minimal for which there exists some $n \geq 1$ such that $\tau^{-m} X_{n}$ is defined but $\tau^{-m} X_{n+1}$ is not. Then $\Gamma$ contains an infinite sectional path

$$
\cdots \rightarrow \tau^{-m+1} X_{i} \rightarrow \cdots \rightarrow \tau^{-m+1} X_{n+1} \rightarrow \tau^{-m+1} X_{n}
$$

having infinitely many arrows of finite global left degree. Setting $Y_{0}=\tau^{-m} X_{n}$ and arguing as in the proof of theorem 5.7, we see that $\Gamma$ contains a sectional path $Y_{t} \rightarrow \cdots \rightarrow Y_{1} \rightarrow Y_{0}$ with $t \geq 1$ and $\tau Y_{1} \neq \tau^{-m+1} X_{n+1}$, and $\mathcal{A}$ has irreducible morphisms $g_{j}: Y_{j} \rightarrow Y_{j-1}$, $h_{j}: \tau Y_{j} \rightarrow \tau Y_{j-1}$, and $f_{j}: \tau Y_{j-1} \rightarrow Y_{j}, j=1, \ldots, t$, such that $\tau Y_{0} \stackrel{f_{1}}{\longrightarrow} Y_{1} \stackrel{g_{1}}{\longrightarrow} Y_{0}$, and

$$
\tau Y_{j} \stackrel{\left(f_{j+1}, h_{j}\right)}{\longrightarrow} Y_{j+1} \amalg \tau Y_{j-1} \stackrel{\left({ }_{f_{j}+1}\right)}{\longrightarrow} Y_{j}, j=1, \ldots, t-1,
$$

and $\tau Y_{t} \stackrel{h_{t}}{\longrightarrow} Y_{1} \stackrel{f_{t}}{\longrightarrow} Y_{0}$ are Auslander-Reiten sequences. In particular, we have $h_{t} \cdots h_{1} f_{1}=0$. Applying first Lemma 6.1 then Lemma 1.3 stated in [10], we get an exact triangle $Y_{0}[-1] \stackrel{h}{\longrightarrow} \tau Y_{0} \stackrel{f_{1}}{\longrightarrow} Y_{1} \stackrel{g_{1}}{\longrightarrow} Y_{0}$ in $\mathcal{A}$. Then $h_{t} \cdots h_{1}$ factors through $Y_{0}[-1]$. By Theorem 2.10, $Y_{0}[-1]=\tau Y_{s}$ for some $0 \leq s \leq t$. Since the shift functor is an automorphism of $\mathcal{A}$, we see that $Y_{1}[-1]$ is the only immediate predecessor of $Y_{0}[-1]$ in $\Gamma$. Hence $s=t$, and $\tau^{2} Y_{t-1}=Y_{1}[-1]$. Now $\Gamma$ contains two sectional paths

$$
\tau^{t+1} Y_{0} \rightarrow \tau^{t} Y_{1} \rightarrow \cdots \rightarrow \tau^{2} Y_{t-1} \rightarrow \tau Y_{t}
$$

and

$$
Y_{t}[-1] \rightarrow Y_{t-1}[-1] \rightarrow \cdots \rightarrow Y_{1}[-1] \rightarrow Y_{0}[-1],
$$

which end with the same arrow. Since $\Gamma$ is smooth, the two paths coincide. In particular, $\tau^{t+1} Y_{0}=Y_{t}[-1]$. This is absurd since $\tau^{t+1} Y_{0}$ has two distinct immediate predecessors $\tau^{t+1} Y_{1}$ and $\tau^{t-m+1} Y_{n+1}$ in $\Gamma$, while $Y_{t}[-1]$ has only one immediate predecessor $\tau Y_{t-1}[-1]$. This establishes the statement. The proof of the theorem is completed.

6.4. Corollary. Let $\mathcal{A}$ be a Krull-Schmidt triangulated R-category which is triangle-connected but not triangle-simple. If $\mathcal{A}$ is an AuslanderReiten category, then every component of $\Gamma_{\mathcal{A}}$ is regular which is either $\tau$-periodic or of shape $\mathbb{Z} \Delta$ where $\Delta$ is a locally finite valued quiver with no oriented cycle. 
ExAMPLE. Let $\Delta$ be a non-trivial Dynkin quiver and $k$ an algebraically closed field. The cluster category $\mathcal{C}$ constructed as a quotient of the bounded derived category $D^{b}(k \Delta)$ of the path algebra $k \Delta$ is a triangulated Auslander-Reiten $k$-category with $\Gamma_{\mathcal{C}} \cong \mathbb{Z} \Delta /\langle\sigma\rangle$, where $\sigma$ is a non-trivial automorphism of $\mathbb{Z} \Delta$; see [9].

We conclude the paper with an application to the bounded derived category of an artin algebra of finite global dimension.

6.5. Theorem. Let $A$ be a connected artin R-algebra. If $A$ is of finite positive global dimension, then the components of $\Gamma_{D^{b}(A)}$ are either stable tubes or of shape $\mathbb{Z} \Delta$ where $\Delta$ is a locally finite valued quiver with no oriented cycle.

Proof. Assume that $A$ is of finite positive global dimension. Then $D^{b}(A)$ is an Auslander-Reiten $R$-category; see [10, (4.6)], which is connected of infinite type as an $R$-category and not triangle-simple as a triangulated category. Let $\Gamma$ be a component of $\Gamma_{D^{b}(A)}$, which is infinite by Lemma 5.1. If $\Gamma$ is $\tau$-periodic, then it is a stable tube by Theorem 5.5(2). Otherwise, by Corollary $6.4, \Gamma \cong \mathbb{Z} \Delta$ where $\Delta$ is a locally finite valued quiver with no oriented cycle. The proof of the theorem is completed.

\section{References}

[1] M. Auslander, "Almost split sequences and algebraic geometry", London Math. Soc. Lecture Note Ser. 116 (Cambridge University Press, Cambridge, 1986) 165 - 179.

[2] M. Auslander, "Isolated sigularities and existence of almost split sequences", Lecture Notes in Mathematics 1178 (Springer, Berlin, 1986) $194-242$.

[3] M. Auslander and I. Reiten, "Representation theory of artin algebras III", Comm. Algebra 3 (1975) 239 - 294.

[4] M. Auslander And I. Reiten, "Representation theory of artin algebras IV", Comm. Algebra 5 (1977) $443-518$.

[5] M. Auslander, I. Reiten and S. O. Smalø, "Representation Theory of Artin Algebras", Cambridge Studies in Advanced Mathematics 36 (Camridge Univeristy Press, Cambridge, 1995).

[6] R. Bautista, "Irreducible morphisms and the radical of a category", Ann. Inst. Math. Univ. Nac. Auto. Mex. 22 (1982) 83 - 135.

[7] R. BAutista And M. Kleiner, "Almost split sequences for relatively projective modules", J. Algebra 135 (1990) 19 - 56. 
[8] R. Bautista And S. O. Smal $\varnothing$, "Non-existent cycles", Comm. Algebra, 11 (1983) 1755 - 1767.

[9] A. B. Buan, R. Marsh, M. Reineke, I. Reiten, G. Todorov, "Tilting theory and cluster combinatorics", Adv. Math. 204 (2006) 572 - 618.

[10] D. HAPPEL, "Triangulated Categories in the Representation Theory of Finite Dimensional Algebras", London Mathematical Society Lecture Notes Series 119 (Cambridge University Press, Cambridge, 1988).

[11] D. Happel, U. Preser, and C. M. Ringel, "Vinberg's charaterization of Dynkin diagrams using subadditive functions with application to DTrperiodic modules", Lecture Notes in Mathematics 832 (Springer, Berlin, 1982) $280-294$.

[12] K. Igusa And G. Todorov, "A characterization of finite AuslanderReiten quivers", J. Algebra 89 (1984) 148 - 177.

[13] O. IyAma, "Higher-dimensional Auslander-Reiten theory on maximal orthogonal subcateogries", Adv. Math. 210 (2007) 22 - 50.

[14] P. Jørgensen, "Auslander-Reiten theory over topological spaces", Comment. Math. Helv. 79 (2004) 160-182.

[15] P. Jørgensen, "Calabi-Yau categories and Poincaré duality spaces", Trends in Representation Theory of Algebras and Related Topics (European Mathematical Society Publishing House, Zürich 2008) 399 - 431.

[16] P. LARRIVÉE, "The Auslander-Reiten quiver of a Krull-Schmidt category," Mémoir de maîtrise (Université de Sherbrooke, Sherbrooke, 1998).

[17] H. Lenzing And J. A. DE la PeÑA, "Wild canonical algebras", Math. Z. 224 (1997) 403 - 425.

[18] S. LIU, "Degrees of irreducible maps and the shapes of Auslander-Reiten quivers," J. London Math. Soc. 45 (1992) 32 - 54.

[19] S. LIU, "Semi-stable components of an Auslander-Reiten quiver," J. London Math. Soc. 47 (1993) 405 - 416.

[20] S. LiU, "Shapes of connected components of the Auslander-Reiten quivers of artin algebras," Representation theory of algebras and related topics (Mexico City, 1994) Canad. Math. Soc. Conf. Proc. 19 (Providence, RI, 1996) $109-137$.

[21] R. Martínez-Villa and D. Zacharia, "Auslander-Reiten sequences, locally free sheaves and Chebysheff polynomials", Compos. Math. 142 (2006) $397-408$.

[22] I. REITEN AND M. VAN DEN BERGH, "Noetherian hereditary abelian categories satisfying Serre duality", J. Amer. Math. Soc. 15 (2002) 295 - 366.

[23] C. M. Ringel, "Finite-dimensional hereditary algebras of wild representation type", Math. Z. 161 (1978) 236 - 255.

[24] C. M. RingEL, "Tame Algebras and Integral Quadratic Forms", Lecture Notes in Mathematics 1099 (Springer-Verlag, Berlin, 1984).

[25] P. J. WeBb, "The Auslander-Reiten quiver of a finite group", Math. Z. 179 (1982) 97 - 121

[26] A. Wiedemann, "Orders with loops in their Auslander-Reiten graph", Comm. Algebra 9 (1980) 641 - 656.

[27] Jie XiaO AND Bin Zhu, "Locally finite triangulated categories", J. Algebra 290 (2005) 473 - 490. 
[28] Y. Zhang, "The structure of stable components", Canad. J. Math. 43 (1991) $652-672$.

São Paulo J.Math.Sci. 4, 3 (2010), 425-472 